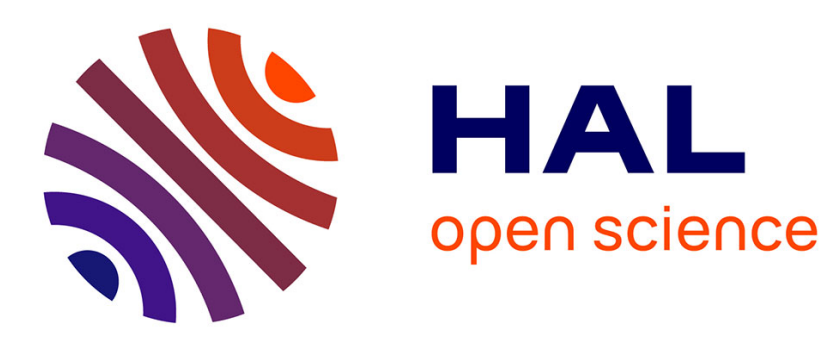

\title{
Stabilization of explicit coupling in fluid-structure interaction involving fluid incompressibility
}

Erik Burman, Miguel Angel Fernández

\section{To cite this version:}

Erik Burman, Miguel Angel Fernández. Stabilization of explicit coupling in fluid-structure interaction involving fluid incompressibility. Computer Methods in Applied Mechanics and Engineering, 2009, 198 (5-8), pp.766-784. 10.1016/j.cma.2008.10.012 . inria-00247409v4

\section{HAL Id: inria-00247409 https://hal.inria.fr/inria-00247409v4}

Submitted on 23 Oct 2008

HAL is a multi-disciplinary open access archive for the deposit and dissemination of scientific research documents, whether they are published or not. The documents may come from teaching and research institutions in France or abroad, or from public or private research centers.
L'archive ouverte pluridisciplinaire HAL, est destinée au dépôt et à la diffusion de documents scientifiques de niveau recherche, publiés ou non, émanant des établissements d'enseignement et de recherche français ou étrangers, des laboratoires publics ou privés. 


\section{N R I A}

INSTITUT NATIONAL DE RECHERCHE EN INFORMATIQUE ET EN AUTOMATIQUE

Stabilization of explicit coupling in fluid-structure interaction involving fluid incompressibility

Erik Burman — Miguel A. Fernández

\section{$N^{\circ} 6445$}

February 2008

Thème BIO

apport

de recherche 



\title{
RINRIA
}

\section{Stabilization of explicit coupling in fluid-structure interaction involving fluid incompressibility}

\author{
Erik Burman^, Miguel A. Fernándeð† \\ Thème BIO - Systèmes biologiques \\ Projet REO \\ Rapport de recherche $\mathrm{n}^{\circ} 6445$ - February 2008 - 41 pages
}

\begin{abstract}
In this work we propose a stabilized explicit coupling scheme for fluid-structure interaction problems involving a viscous incompressible fluid. The coupled discrete formulation is based on Nitsche's method with a time penalty term giving $L^{2}$-control on the fluid pressure variations at the interface. The scheme is stable, in the energy norm, irrespectively of the fluid-structure density ratio. Numerical experiments, in two and three dimensions, show that optimal time accuracy can be obtained by performing a few defect-correction iterations.
\end{abstract}

Key-words: Fluid-structure interaction, Nitsche's method, fluid incompressibility, time discretization, explicit coupling, loosely coupled schemes, defectcorrection method

Preprint accepted for publication in Computer Methods in Applied Mechanics and Engineering.

* University of Sussex, UK; e-mail: E.N.Burman@sussex.ac.uk

$\dagger$ INRIA, REO team; e-mail: Miguel.Fernandez@inria.fr 


\section{Couplage explicite stabilisé en interaction fluide-structure avec fluide incompressible}

Résumé : Dans ce travail on propose un schéma de couplage explicite pour la résolution numérique de problémes d'interaction fluide-structure comportant un fluide visqueux incompressible. La formulation discrète couplée est bassée dans la méthode de Nitsche et le rajout d'un terme de pénalisation donnant un contrôle $L^{2}$ de la variation de la pression du fluide sur l'interface. Le schéma est stable, dans la norme de l'énergie, indépendamment du rapport de densités fluide-structure. Des expériences numériques, en deux et trois dimensions, montrent qu'on peut obtenir une précision optimale en temps après quelques itérations d'un algorithme de Résidus Corrigés.

Mots-clés : Interaction fluide-structure, méthode de Nitsche, fluide incompressible, discrétisation en temps, couplage explicite, algorithme de Résidus Corrigés 


\section{Introduction}

Computational Fluid-Structure Dynamics (CFSD) is of great importance in practically all engineering fields, from aeroelasticity to bio-mechanics problems (see, for instance, [28, 10, 11, 24, 30, 27, 32, 17, 14, 38, 2] and the references therein).

In this paper we address the numerical simulation of fluid-structure interaction problems involving a viscous incompressible fluid. This problem is particularly difficult to treat efficiently when the fluid added-mass, acting on the structure, is significant. In other words, when the fluid and solid densities are close or when the domain is slender (see e.g. [7]). Indeed, in such situations, explicit coupling (or loosely coupled) schemes, i.e. that only involve the solution of the fluid and the structure once (or just a few times) per time step (see [31, 33, 11] for instance), are known to give rise to numerical instabilities (first reported in [26], see also [24, 30]). Theoretical explanations of this issue have been reported in [7] (see also [16]). In particular, in [16], it is argued that no explicit scheme can be constructed which would be unconditionally stable with respect to the fluid-structure density ratio.

Up to now, these instabilities have been overcome mainly through the use of implicit coupling (or strongly coupled) schemes (see [26, 17, 13, 14, 8, 1] and the references therein). Such an approach leads to a fully coupled problem at each time step, the solution of which often requires a huge computational effort. Recent advances suggest the use of semi-implicit coupling schemes [12 (see also [34), which involve a simplified fully coupled problem. Note that a critical added-mass effect can also compromise the efficiency of the iterative procedure used for solving the implicit component of the coupling scheme (see [7, 1]). Although significant improvements have been achieved in the last years, to the authors knowledge, none of the existing strategies is able to allow fully explicit coupling without compromising stability.

In this paper, we propose a stabilized explicit coupling scheme, whose stability properties are independent of the added-mass effect. The coupling interface conditions are treated in a weak sense, using a formulation based on Nitsche's method $29,3,20$. Note that an explicit decoupling of the fluid and the structure, using the Nitsche's formulation alone, is not stable irrespectively of the added-mass effect. The key ingredient, to obtain such an added-mass uniform stability, is the time penalty term on the fluid pressure fluctuations we propose to add on the interface.

The method may suffer from a deterioration of the time accuracy, due to the the weak consistency of the time penalty stabilization term, which rates as $O\left(\delta t^{\frac{1}{2}}\right)$. In order to recover optimal accuracy, we propose an improved explicit coupling scheme involving a few defect-correction iterations (see e.g. [37]). Numerical experiments, in the linear and non-linear case, show that optimal time accuracy can be obtained by performing one defect-correction iteration (when first order accuracy is expected for the underlying implicit coupling scheme).

The remainder of the paper is organized as follows. In the next section, we consider a simplified (linear) fluid-structure interaction problem based on the coupling of a linear elastic solid and a Stokes-flow. This linear framework will be the common starting-point for the different coupling strategies introduced in this paper. The corresponding space semi-discretized Nitsche's formulation is described in section 83 . Section $\$ 4$ is devoted to the time-discretization. 
In particular, in paragraphs $\$ 4.1$ and $\$ 4.2$, we introduce the classical coupling schemes, implicit and explicit, and we analyze their stability properties within the Nitsche's framework. Motivated by these results, in section \$5 we introduce the stabilized explicit coupling scheme and we establish its enhanced stability features. A formal consistency analysis, that highlights the optimality loss due to the added stabilizing term, is performed in paragraph $\$ 5.3$. We propose to recover optimal time-accuracy by performing a few defect-correction iterations. The resulting coupling algorithm is given in paragraph 5.4 and a non-linear variant in paragraph $\$ 5.5$. Finally, section 86 is devoted to the numerical tests, in the linear and non-linear case, confirming the theoretical results and illustrating the efficiency and robustness of the proposed approaches. A summary of the results and some conclusions are given in section $\$ 7$

Some preliminary results of this work have been announced, without proof, in [4].

\section{A simplified coupled problem}

In order to facilitate the analysis and to motivate de different coupling schemes introduced in this paper, we first consider a low Reynolds regime and assume that the interface undergoes infinitesimal displacements. The fluid is described

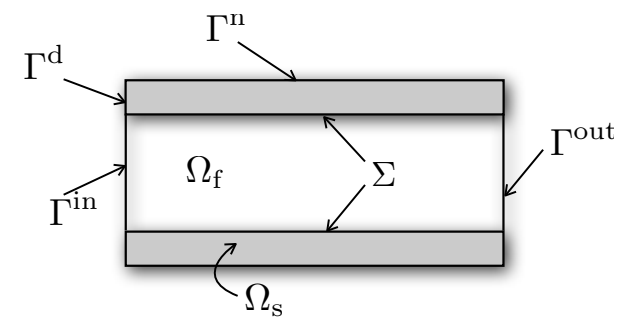

Figure 1: Geometrical description

by the Stokes equations, in a fixed domain $\Omega_{\mathrm{f}} \subset \mathbb{R}^{d}, d=2,3$, and the structure by the classical linear elasticity equations, in the solid domain $\Omega_{\mathrm{s}} \subset \mathbb{R}^{d}$. We denote by $\Sigma \stackrel{\text { def }}{=} \partial \Omega_{\mathrm{s}} \cap \partial \Omega_{\mathrm{f}}$ the fluid-structure interface and $\partial \Omega_{\mathrm{f}}=\Gamma^{\text {in }} \cup \Gamma^{\text {out }} \cup \Sigma$, $\partial \Omega_{\mathrm{s}}=\Gamma^{\mathrm{d}} \cup \Gamma^{\mathrm{n}} \cup \Sigma$, are given partitions of the fluid and solid boundaries, respectively (see Figure 1). Our simplified coupled problem reads as follows: Find the fluid velocity $\boldsymbol{u}: \Omega_{\mathrm{f}} \times \mathbb{R}^{+} \rightarrow \mathbb{R}^{d}$, the fluid pressure $p: \Omega_{\mathrm{f}} \times \mathbb{R}^{+} \rightarrow \mathbb{R}$ and the structure displacement $\boldsymbol{\eta}: \Omega_{\mathrm{s}} \times \mathbb{R}^{+} \rightarrow \mathbb{R}^{d}$ such that

$$
\begin{gathered}
\left\{\begin{aligned}
\rho_{\mathrm{f}} \partial_{t} \boldsymbol{u}-\boldsymbol{\nabla} \cdot \boldsymbol{\sigma}(\boldsymbol{u}, p)=\mathbf{0}, & \text { in } \Omega_{\mathrm{f}}, \\
\boldsymbol{\nabla} \cdot \boldsymbol{u}=0, & \text { in } \Omega_{\mathrm{f}}, \\
\boldsymbol{u}=\overline{\boldsymbol{u}}, & \text { on } \Gamma^{\text {in }}, \\
\boldsymbol{\sigma}(\boldsymbol{u}, p) \boldsymbol{n}=\boldsymbol{g}, & \text { on } \Gamma^{\text {out }},
\end{aligned}\right. \\
\left\{\begin{aligned}
\rho_{\mathrm{s}} \partial_{t t}^{2} \boldsymbol{\eta}-\nabla \cdot \boldsymbol{\sigma}_{\mathrm{s}}(\boldsymbol{\eta})=\mathbf{0}, & \text { in } \quad \Omega_{\mathrm{s}}, \\
\boldsymbol{\eta}=\mathbf{0}, & \text { on } \quad \Gamma^{\mathrm{d}}, \\
\boldsymbol{\sigma}_{\mathrm{s}}(\boldsymbol{\eta}) \boldsymbol{n}_{\mathrm{s}}=\mathbf{0}, & \text { on } \quad \Gamma^{\mathrm{n}}
\end{aligned}\right.
\end{gathered}
$$


satisfying the interface coupling conditions

$$
\left\{\begin{aligned}
\boldsymbol{u} & =\partial_{t} \boldsymbol{\eta}, & & \text { on } \quad \Sigma, \\
\boldsymbol{\sigma}_{\mathrm{s}}(\boldsymbol{\eta}) \boldsymbol{n}_{\mathrm{s}} & =-\boldsymbol{\sigma}(\boldsymbol{u}, p) \boldsymbol{n}, & & \text { on } \quad \Sigma,
\end{aligned}\right.
$$

and the initial conditions $\boldsymbol{u}(0)=\boldsymbol{u}_{0}, \boldsymbol{\eta}(0)=\boldsymbol{\eta}_{0}$ and $\partial_{t} \boldsymbol{\eta}(0)=\boldsymbol{v}_{0}$. Here, $\rho_{\mathrm{f}}$ and $\rho_{\mathrm{s}}$ stand for the fluid and solid densities, $\boldsymbol{\sigma}(\boldsymbol{u}, p) \stackrel{\text { def }}{=}-p \boldsymbol{I}+2 \mu \boldsymbol{\epsilon}(\boldsymbol{u})$ and $\boldsymbol{\sigma}_{\mathrm{s}}(\boldsymbol{\eta})$ for the fluid and solid stress tensors, $\mu$ for the fluid dynamic viscosity, $\boldsymbol{\epsilon}(\boldsymbol{u}) \stackrel{\text { def }}{=} \frac{1}{2}\left(\boldsymbol{\nabla} \boldsymbol{u}+\boldsymbol{\nabla} \boldsymbol{u}^{\mathrm{T}}\right)$ for the fluid strain rate tensor, $\overline{\boldsymbol{u}}$ for a given velocity profile, $\boldsymbol{g}$ for a given surface load and $\boldsymbol{n}, \boldsymbol{n}_{\mathrm{s}}$ for the exterior unit normal vectors on the boundary of $\Omega_{\mathrm{f}}$ and $\Omega_{\mathrm{s}}$, respectively.

Although (1)-(3) is a simplified linear coupled model, it features some of the main stability issues that appear in complex non-linear fluid-structure interaction problems involving a viscous incompressible fluid (see e.g. [24, 30, 7, 12]). Extensions to the non-linear case of some of the coupling strategies introduced in this paper are provided in paragraph 5.5

\section{Nitsche's formulation: space semi-discretization}

In this section, we provide a space semi-discretized formulation of the coupled problem (1)-(3). The fluid and structure equations (1)-(2) are discretized using standard finite elements techniques in both domains. The coupling conditions (3) are treated in a less standard fashion: by using Nitsche's penalty method.

Originally, Nitsche's method [29] (see [18] for a review) is a method for imposing essential boundary conditions weakly. Unlike the penalty method, it is consistent with the original differential equation. Indeed, optimal convergence is retained without perturbing the conditioning of the matrix. Recently, Nitsche's method was proposed in a domain decomposition framework in [3]. It has then been extended to different multi-physics problems. Let us cite the coupling of the Stokes-Darcy problem [5], the coupling of elliptic-hyperbolic problems or problems with discontinuous diffusivities [6]. In the context of fluid-structure interaction, using implicit coupling, some results are given for vibration problems (acoustics) in [19] and for transient fluid-structure interaction problems with moving fluid domains in [20].

In what follows, we will consider the usual Sobolev's spaces $W^{m, q}(\Omega)$, with norm $\|\cdot\|_{m, q, \Omega}, m \geq 0$ and $q \geq 1$. In particular, we have $L^{q}(\Omega)=W^{0, q}(\Omega)$. We use the standard notation $H^{m}(\Omega) \stackrel{\text { def }}{=} W^{m, 2}(\Omega)$. The norm of $H^{m}(\Omega)$ is denoted by $\|\cdot\|_{m, \Omega}$, in particular $\|\cdot\|_{0, \Omega}$ stands for the norm of $L^{2}(\Omega)$. Moreover, for each $X \subset \partial \Omega$, with meas $(X)>0$, we define $H_{X}^{1}(\Omega)$ as the space of functions $v \in H^{1}(\Omega)$ such that $v_{\mid X}=0$ in the sense of traces.

For the discretization in space, we introduce a family $\left\{\mathcal{I}_{\mathrm{f}, h}\right\}_{h>0}\left(\right.$ resp. $\left.\left\{\mathcal{T}_{\mathrm{s}, h}\right\}_{h>0}\right)$ of regular finite element triangulations of the domain $\Omega_{\mathrm{f}}\left(\operatorname{resp} . \Omega_{\mathrm{s}}\right)$. The subscript $h$ refers to the level of refinement of the triangulations. Accordingly, let $W_{h} \times Q_{h}$ denote a conforming, inf-sup stable, finite element approximation of $\left[H^{1}\left(\Omega_{\mathrm{f}}\right)\right]^{d} \times L^{2}\left(\Omega_{\mathrm{f}}\right)$, and $X_{h}$ a conforming finite element approximation of $\left[H_{\Gamma^{\mathrm{d}}}^{1}\left(\Omega_{\mathrm{s}}\right)\right]^{d}$. We also introduce the space $V_{h} \stackrel{\text { def }}{=} W_{h} \cap\left[H_{\Gamma^{\text {in }}}^{1}\left(\Omega_{\mathrm{f}}\right)\right]^{d}$.

We may write the space semi-discretized Nitsche's formulation of (1)-(3) as: For all $t>0$, find $\left(\boldsymbol{u}_{h}, p_{h}, \boldsymbol{\eta}_{h}, \dot{\boldsymbol{\eta}}_{h}\right) \in W_{h} \times Q_{h} \times X_{h} \times X_{h}$, with $\boldsymbol{u}_{h}=\overline{\boldsymbol{u}}_{h}$ on $\Gamma^{\text {in }}$, 
such that

$$
\begin{aligned}
& A_{\mathrm{f}}\left(\left(\boldsymbol{u}_{h}, p_{h}\right),\left(\boldsymbol{v}_{h}, q_{h}\right)\right)+ A_{\mathrm{s}}\left(\left(\boldsymbol{\eta}_{h}, \dot{\boldsymbol{\eta}}_{h}\right),\left(\boldsymbol{w}_{h}, \dot{\boldsymbol{w}}_{h}\right)\right) \\
&-\int_{\Sigma} \boldsymbol{\sigma}\left(\boldsymbol{u}_{h}, p_{h}\right) \boldsymbol{n} \cdot\left(\boldsymbol{v}_{h}-\boldsymbol{w}_{h}\right)-\int_{\Sigma}\left(\boldsymbol{u}_{h}-\partial_{t} \boldsymbol{\eta}_{h}\right) \cdot \boldsymbol{\sigma}\left(\alpha \boldsymbol{v}_{h},-q_{h}\right) \boldsymbol{n} \\
&+\gamma \frac{\mu}{h} \int_{\Sigma}\left(\boldsymbol{u}_{h}-\partial_{t} \boldsymbol{\eta}_{h}\right) \cdot\left(\boldsymbol{v}_{h}-\boldsymbol{w}_{h}\right)=\int_{\Gamma_{\text {out }}} \boldsymbol{g} \cdot \boldsymbol{v}_{h},
\end{aligned}
$$

for all $\left(\boldsymbol{v}_{h}, q_{h}, \boldsymbol{w}_{h}, \dot{\boldsymbol{w}}_{h}\right) \in V_{h} \times Q_{h} \times X_{h} \times X_{h}$. Here, $\overline{\boldsymbol{u}}_{h}$ stands for a suitable interpolation of the boundary data $\overline{\boldsymbol{u}}$ on $\Gamma^{\text {in }}, \alpha$ for a given parameter taking values in $\{-1,1\}$, and $\gamma>0$ for the Nitsche's penalty parameter (dimensionless). Moreover, the fluid and solid (volume) bilinear forms are given by

$$
\begin{aligned}
A_{\mathrm{f}}\left(\left(\boldsymbol{u}_{h}, p_{h}\right),\left(\boldsymbol{v}_{h}, q_{h}\right)\right) \stackrel{\text { def }}{=} & \rho_{\mathrm{f}} \\
\int_{\Omega_{\mathrm{f}}} & \partial_{t} \boldsymbol{u}_{h} \cdot \boldsymbol{v}_{h}+\int_{\Omega_{\mathrm{f}}} \boldsymbol{\sigma}\left(\boldsymbol{u}_{h}, p_{h}\right): \boldsymbol{\epsilon}\left(\boldsymbol{v}_{h}\right) \\
& +\int_{\Omega_{\mathrm{f}}} q_{h} \boldsymbol{\nabla} \cdot \boldsymbol{u}_{h}, \\
A_{\mathrm{s}}\left(\left(\boldsymbol{\eta}_{h}, \dot{\boldsymbol{\eta}}_{h}\right),\left(\boldsymbol{w}_{h}, \dot{\boldsymbol{w}}_{h}\right)\right) \stackrel{\text { def }}{=} & \rho_{\mathrm{s}} \int_{\Omega_{\mathrm{s}}} \partial_{t} \dot{\boldsymbol{\eta}}_{h} \cdot \boldsymbol{w}_{h}+a_{\mathrm{s}}\left(\boldsymbol{\eta}_{h}, \boldsymbol{w}_{h}\right) \\
& +\rho_{\mathrm{s}} \int_{\Omega_{\mathrm{s}}}\left(\dot{\boldsymbol{\eta}}_{h}-\partial_{t} \boldsymbol{\eta}_{h}\right) \cdot \dot{\boldsymbol{w}}_{h},
\end{aligned}
$$

where $a_{\mathrm{s}}$ stands for a general solid stiffness symmetric bilinear form.

Note the boundary integrations on $\Sigma$, these are the Nitsche's weak coupling terms. The interface integrals involving the stress tensor $\boldsymbol{\sigma}$ are computed facewise, as broken integrals. Let us emphasize that the approximation in each sub-domain (fluid and structure) involves standard conforming finite element spaces, with strongly imposed Dirichlet boundary conditions on $\Gamma^{\text {in }}$ and $\Gamma^{\mathrm{d}}$. Only the interface coupling conditions (3) are treated using Nitsche's method. In particular, the functions of $V_{h}$ and $X_{h}$ do not necessarily match at the interface $\Sigma$, which leads to the interface integrals in (4). This differs from classic fluidstructure formulations (see e.g. 24]), where interface integrals cancel due to the strong enforcement of the kinematic condition $(3)_{1}$.

Remark 3.1 The parameter $\alpha$ characterizes the type of formulation: symmetric $(\alpha=1)$ or non-symmetric $(\alpha=-1)$. The former is often preferred, for instance, in order to derive optimal $L^{2}$-error estimates for the fluid velocity (using the Aubin-Nitsche's duality trick). Note that the Nitsche's formulation (4) differs from the one proposed in [20], within a space-time framework. Indeed, in [20], the symmetrizing (consistent) coupling term is given by $\int_{\Sigma}\left(\boldsymbol{u}_{h}-\partial_{t} \boldsymbol{\eta}_{h}\right) \cdot \boldsymbol{\sigma}\left(\boldsymbol{v}_{h}, q_{h}\right) \boldsymbol{n}$, instead of $\int_{\Sigma}\left(\boldsymbol{u}_{h}-\partial_{t} \boldsymbol{\eta}_{h}\right) \cdot \boldsymbol{\sigma}\left(\alpha \boldsymbol{v}_{h},-q_{h}\right) \boldsymbol{n}$, as we propose in (4).

Remark 3.2 As noticed in [3, 18], the first coupling term in [4] 3 , involving the fluid stresses on the interface, could be replaced by any convex combination of the fluid and solid interface stresses, namely,

$$
\beta \boldsymbol{\sigma}\left(\boldsymbol{u}_{h}, p_{h}\right) \boldsymbol{n}+(1-\beta) \boldsymbol{\sigma}_{\mathrm{s}}\left(\boldsymbol{\eta}_{h}\right) \boldsymbol{n}, \quad \beta \in[0,1] .
$$

Here, for stability purposes (see Section 5), we have chosen a fluid-sided "mortaring", i.e. we take $\beta=1$. 


\subsection{Stability}

In what follows, we shall make use of the following local trace-inverse inequality (see e.g. [39])

$$
\left\|\boldsymbol{v}_{h}\right\|_{0, \partial K}^{2} \leq C_{\mathrm{TI}} h^{-1}\left\|\boldsymbol{v}_{h}\right\|_{0, K}^{2}, \quad \forall \boldsymbol{v}_{h} \in W_{h}
$$

for all $K \in \mathcal{T}_{\text {f,h }}$.

The following Lemma provides an a priori energy stability estimate for the semi-discrete formulation (4).

Lemma 3.3 Assume that the fluid-structure system is isolated, i.e. $\overline{\boldsymbol{u}}=\mathbf{0}$ and $\boldsymbol{g}=\mathbf{0}$. Let $\left(\boldsymbol{u}_{h}, p_{h}, \boldsymbol{\eta}_{h}, \dot{\boldsymbol{\eta}}_{h}\right)$ be the solution of (4). Then, under the condition

$$
\gamma>4(1+\alpha)^{2} C_{\mathrm{TI}},
$$

the following energy estimate holds,

$$
\begin{aligned}
& \frac{\rho_{\mathrm{f}}}{2}\left\|\boldsymbol{u}_{h}\right\|_{0, \Omega_{\mathrm{f}}}^{2}+\frac{\rho_{\mathrm{s}}}{2}\left\|\dot{\boldsymbol{\eta}}_{h}\right\|_{0, \Omega_{\mathrm{s}}}^{2}+\frac{1}{2} a_{\mathrm{s}}\left(\boldsymbol{\eta}_{h}, \boldsymbol{\eta}_{h}\right)+\frac{3 \mu}{4} \int_{0}^{t}\left\|\boldsymbol{\epsilon}\left(\boldsymbol{u}_{h}\right)\right\|_{0, \Omega_{\mathrm{f}}}^{2} \\
& +\frac{3}{4} \frac{\gamma \mu}{h} \int_{0}^{t}\left\|\boldsymbol{u}_{h}-\partial_{t} \boldsymbol{\eta}_{h}\right\|_{0, \Sigma}^{2} \leq \frac{\rho_{\mathrm{f}}}{2}\left\|\boldsymbol{u}_{h}^{0}\right\|_{0, \Omega_{\mathrm{f}}}^{2}+\frac{\rho_{\mathrm{s}}}{2}\left\|\dot{\boldsymbol{\eta}}_{h}^{0}\right\|_{0, \Omega_{\mathrm{s}}}^{2}+\frac{1}{2} a_{\mathrm{s}}\left(\boldsymbol{\eta}_{h}^{0}, \boldsymbol{\eta}_{h}^{0}\right) .
\end{aligned}
$$

In particular, for $\alpha=-1$ this estimate holds true for all values of the Nitsche's penalty parameter $\gamma>0$.

Proof. By taking

$$
\left(\boldsymbol{v}_{h}, q_{h}, \boldsymbol{w}_{h}, \dot{\boldsymbol{w}}_{h}\right)=\left(\boldsymbol{u}_{h}, p_{h}, \partial_{t} \boldsymbol{\eta}_{h}, \partial_{t} \dot{\boldsymbol{\eta}}_{h}\right),
$$

in (4) we have,

$$
\begin{aligned}
\frac{\mathrm{d}}{\mathrm{d} t}\left(\frac{\rho_{\mathrm{f}}}{2}\left\|\boldsymbol{u}_{h}\right\|_{0, \Omega_{\mathrm{f}}}^{2}+\frac{\rho_{\mathrm{s}}}{2}\left\|\dot{\boldsymbol{\eta}}_{h}\right\|_{0, \Omega_{\mathrm{s}}}^{2}+\frac{1}{2} a_{\mathrm{s}}\left(\boldsymbol{\eta}_{h}, \boldsymbol{\eta}_{h}\right)\right)+2 \mu\left\|\boldsymbol{\epsilon}\left(\boldsymbol{u}_{h}\right)\right\|_{0, \Omega_{\mathrm{f}}}^{2} \\
+\frac{\gamma \mu}{h}\left\|\boldsymbol{u}_{h}-\partial_{t} \boldsymbol{\eta}_{h}\right\|_{0, \Sigma}^{2}-2(1+\alpha) \mu \int_{\Sigma} \boldsymbol{\epsilon}\left(\boldsymbol{u}_{h}\right) \boldsymbol{n} \cdot\left(\boldsymbol{u}_{h}-\partial_{t} \boldsymbol{\eta}_{h}\right)=0 .
\end{aligned}
$$

On the other hand, using the local trace-inverse inequality (5) and after applying Young's inequality, we obtain

$$
\begin{aligned}
2(1+\alpha) \mu \int_{\Sigma} \boldsymbol{\epsilon}\left(\boldsymbol{u}_{h}\right) \boldsymbol{n} \cdot\left(\boldsymbol{u}_{h}-\partial_{t} \boldsymbol{\eta}_{h}\right) \leq & \frac{4(1+\alpha)^{2} \mu C_{\mathrm{TI}}}{\gamma}\left\|\boldsymbol{\epsilon}\left(\boldsymbol{u}_{h}\right)\right\|_{0, \Omega_{\mathrm{f}}}^{2} \\
& +\frac{\gamma \mu}{4 h}\left\|\boldsymbol{u}_{h}-\partial_{t} \boldsymbol{\eta}_{h}\right\|_{0, \Sigma}^{2} .
\end{aligned}
$$

Thus, inserting this expression in (8) and after integration over $(0, t)$, we have

$$
\begin{aligned}
& \frac{\rho_{\mathrm{f}}}{2}\left\|\boldsymbol{u}_{h}(t)\right\|_{0, \Omega_{\mathrm{f}}}^{2}+\frac{\rho_{\mathrm{s}}}{2}\left\|\dot{\boldsymbol{\eta}}_{h}\right\|_{0, \Omega_{\mathrm{s}}}^{2}+\frac{1}{2} a_{\mathrm{s}}\left(\boldsymbol{\eta}_{h}, \boldsymbol{\eta}_{h}\right)+\mu_{\alpha} \int_{0}^{t}\left\|\boldsymbol{\epsilon}\left(\boldsymbol{u}_{h}\right)\right\|_{0, \Omega_{\mathrm{f}}}^{2} \\
& \quad+\frac{3}{4} \frac{\gamma \mu}{h} \int_{0}^{t}\left\|\boldsymbol{u}_{h}-\partial_{t} \boldsymbol{\eta}_{h}\right\|_{0, \Sigma}^{2} \leq \frac{\rho_{\mathrm{f}}}{2}\left\|\boldsymbol{u}_{h}^{0}\right\|_{0, \Omega_{\mathrm{f}}}^{2}+\frac{\rho_{\mathrm{s}}}{2}\left\|\dot{\boldsymbol{\eta}}_{h}^{0}\right\|_{0, \Omega_{\mathrm{s}}}^{2}+\frac{1}{2} a_{\mathrm{s}}\left(\boldsymbol{\eta}_{h}^{0}, \boldsymbol{\eta}_{h}^{0}\right),
\end{aligned}
$$

with

$$
\mu_{\alpha} \stackrel{\text { def }}{=} \mu\left(2-\frac{4(1+\alpha)^{2} C_{\mathrm{TI}}}{\gamma}\right),
$$

which completes the proof. $\square$

Note that the (additional) interface term in the energy inequality (7) appears due to the dissipative character of the Nitsche's coupling. 


\subsection{Partitioned formulation}

In this paper we focus on partitioned procedures for the numerical resolution of the monolithic (or global) formulation (4), i.e. methods which only involve separate solutions of the fluid and structure sub-problems (1) and (2) (with appropriate boundary conditions on $\Sigma$ ). Such procedures enable the design of efficient fluid-structure solution algorithms, while keeping state-of-the-art methods and software in each sub-domain (see e.g. [31, 11, 27, 17, 13, 14, 12]).

To this aim, we reformulate problem (4) in terms of two coupled problems. This can be achieved by simply taking $\left(\boldsymbol{v}_{h}, q_{h}\right)=(\mathbf{0}, 0)$ or $\boldsymbol{w}_{h}=\dot{\boldsymbol{w}}_{h}=\mathbf{0}$ in (4), which yields:

- Solid sub-problem: Given $\left(\boldsymbol{u}_{h}, p_{h}\right) \in W_{h} \times Q_{h}$, find $\left(\boldsymbol{\eta}_{h}, \dot{\boldsymbol{\eta}}_{h}\right) \in X_{h} \times X_{h}$ such that

$A_{\mathrm{S}}\left(\left(\boldsymbol{\eta}_{h}, \dot{\boldsymbol{\eta}}_{h}\right),\left(\boldsymbol{w}_{h}, \dot{\boldsymbol{w}}_{h}\right)\right)+\gamma \frac{\mu}{h} \int_{\Sigma} \partial_{t} \boldsymbol{\eta}_{h} \cdot \boldsymbol{w}_{h}=\gamma \frac{\mu}{h} \int_{\Sigma} \boldsymbol{u}_{h} \cdot \boldsymbol{w}_{h}-\int_{\Sigma} \boldsymbol{\sigma}\left(\boldsymbol{u}_{h}, p_{h}\right) \boldsymbol{n} \cdot \boldsymbol{w}_{h}$,

for all $\left(\boldsymbol{w}_{h}, \dot{\boldsymbol{w}}_{h}\right) \in X_{h} \times X_{h}$.

- Fluid sub-problem: Given $\partial_{t} \boldsymbol{\eta}_{h} \in X_{h}$, find $\left(\boldsymbol{u}_{h}, p_{h}\right) \in W_{h} \times Q_{h}$, with $\boldsymbol{u}_{h}=\overline{\boldsymbol{u}}_{h}$ on $\Gamma^{\mathrm{in}}$, such that

$$
\begin{gathered}
A_{\mathrm{f}}\left(\left(\boldsymbol{u}_{h}, p_{h}\right),\left(\boldsymbol{v}_{h}, q_{h}\right)\right)-\int_{\Sigma} \boldsymbol{\sigma}\left(\boldsymbol{u}_{h}, p_{h}\right) \boldsymbol{n} \cdot \boldsymbol{v}_{h}-\int_{\Sigma} \boldsymbol{u}_{h} \cdot \boldsymbol{\sigma}\left(\alpha \boldsymbol{v}_{h},-q_{h}\right) \boldsymbol{n}+\gamma \frac{\mu}{h} \int_{\Sigma} \boldsymbol{u}_{h} \cdot \boldsymbol{v}_{h} \\
=-\int_{\Sigma} \partial_{t} \boldsymbol{\eta}_{h} \cdot \boldsymbol{\sigma}\left(\alpha \boldsymbol{v}_{h},-q_{h}\right) \boldsymbol{n}+\gamma \frac{\mu}{h} \int_{\Sigma} \partial_{t} \boldsymbol{\eta}_{h} \cdot \boldsymbol{v}_{h}+\int_{\Gamma \text { out }} \boldsymbol{g} \cdot \boldsymbol{v}_{h},
\end{gathered}
$$

for all $\left(\boldsymbol{v}_{h}, q_{h}\right) \in V_{h} \times Q_{h}$.

From the numerical point of view, the discrete solid sub-problem 10 corresponds to the finite element approximation of the structural mechanics subproblem $22_{1}$, with the following Robin-type boundary conditions on the interface:

$$
\boldsymbol{\sigma}_{\mathrm{s}}(\boldsymbol{\eta}) \boldsymbol{n}-\gamma \frac{\mu}{h} \partial_{t} \boldsymbol{\eta}=\boldsymbol{\sigma}(\boldsymbol{u}, p) \boldsymbol{n}-\gamma \frac{\mu}{h} \boldsymbol{u}, \quad \text { on } \quad \Sigma .
$$

On the other hand, the fluid sub-problem (11) is nothing but the finite element approximation of the fluid mechanics sub-problem (1), with the following Dirichlet condition

$$
\boldsymbol{u}=\partial_{t} \boldsymbol{\eta}, \quad \text { on } \Sigma,
$$

weakly enforced using Nitsche's method.

In summary, the fluid-structure Nitsche's formulation (4) can be reformulated, in a partitioned fashion, as two sub-problems (structure and fluid) coupled through Robin, (12), and Dirichlet-Nitsche, (13), transmission conditions. This is in contrast with the traditional Dirichlet-Neumann formulation, where only forces are transferred from the fluid to the solid (see e.g. [24, 25, 17, 14). We refer also to the recent work 1, where the coupling is prescribed in terms of Robin-Robin conditions, in order to accelerate the convergence of a partitioned solution procedure (within the context of an implicit coupling scheme).

Remark 3.4 In the traditional fluid-structure formulations (in which $V_{h}$ and $X_{h}$ match at the interface, up to interpolation), stability requires (at least theoretically, see e.g. [24]) the interface fluid force to be given as the fluid-subproblem 
variational residual. In the solid-subproblem 10 of the Nitsche's formulation (4), on the contrary, no such a variational consistency is needed to ensure stability. The interface fluid force is simply given as an interface integral. This will be crucial, in section $\$ 5$, to stabilize a fluid-structure explicit coupling scheme.

\section{Time discretization: coupling strategies}

For the time discretization we propose to replace all time derivatives in 10 (11) by backward differences. Let $(0, T)$ be the time interval of interest. For a given integer $N \in \mathbb{N}^{*}$, we introduce the time-step $\delta t \stackrel{\text { def }}{=} T / N$ and the time grid $t_{n} \stackrel{\text { def }}{=} n \delta t$, with $0 \leq n \leq N$. In what follows, we will use the following general notation for the first order backward difference $\partial_{\delta t} X^{n+1} \stackrel{\text { def }}{=} \delta t^{-1}\left(X^{n+1}-X^{n}\right)$.

We consider a first order backward difference discretization in the fluid and a Newmark's scheme for the structure, so that the fully discrete fluid and solid bilinear forms at time level $0 \leq n \leq N-1$ are given by:

$$
\begin{aligned}
& A_{\mathrm{f}, \delta t}\left(\left(\boldsymbol{u}_{h}^{n+1}, p_{h}^{n+1}\right),\left(\boldsymbol{v}_{h}, q_{h}\right)\right) \stackrel{\text { def }}{=} \rho_{\mathrm{f}} \int_{\Omega_{\mathrm{f}}} \partial_{\delta t} \boldsymbol{u}_{h}^{n+1} \cdot \boldsymbol{v}_{h}+\int_{\Omega_{\mathrm{f}}} \boldsymbol{\sigma}\left(\boldsymbol{u}_{h}^{n+1}, p_{h}^{n+1}\right): \boldsymbol{\epsilon}\left(\boldsymbol{v}_{h}\right) \\
&+\int_{\Omega_{\mathrm{f}}} q_{h} \boldsymbol{\nabla} \cdot \boldsymbol{u}_{h}^{n+1}, \\
& A_{\mathrm{s}, \delta t}\left(\left(\boldsymbol{\eta}_{h}^{n+1}, \dot{\boldsymbol{\eta}}_{h}^{n+1}\right),\left(\boldsymbol{w}_{h}, \dot{\boldsymbol{w}}_{h}\right)\right) \stackrel{\text { def }}{=} \rho_{\mathrm{s}} \int_{\Omega_{\mathrm{s}}} \partial_{\delta t} \dot{\boldsymbol{\eta}}_{h}^{n+1} \cdot \boldsymbol{w}_{h}+\frac{1}{2} a_{\mathrm{s}}\left(\boldsymbol{\eta}_{h}^{n+1}+\boldsymbol{\eta}_{h}^{n}, \boldsymbol{w}_{h}\right) \\
&+\rho_{\mathrm{s}} \int_{\Omega_{\mathrm{s}}}\left(\frac{\dot{\boldsymbol{\eta}}_{h}^{n+1}+\dot{\boldsymbol{\eta}}_{h}^{n}}{2}-\partial_{\delta t} \boldsymbol{\eta}_{h}^{n+1}\right) \cdot \dot{\boldsymbol{w}}_{h} .
\end{aligned}
$$

As a result, the fully discrete partitioned fluid-structure formulation is given by:

- Solid sub-problem: Given $\left(\boldsymbol{u}_{h}^{*}, p_{h}^{*}\right) \in W_{h} \times Q_{h}$, find $\left(\boldsymbol{\eta}_{h}^{n+1}, \dot{\boldsymbol{\eta}}_{h}^{n+1}\right) \in$ $X_{h} \times X_{h}$ such that

$$
\begin{aligned}
A_{\mathrm{s}, \delta t}\left(\left(\boldsymbol{\eta}_{h}^{n+1}, \dot{\boldsymbol{\eta}}_{h}^{n+1}\right),\left(\boldsymbol{w}_{h}, \dot{\boldsymbol{w}}_{h}\right)\right)+\gamma \frac{\mu}{h} \int_{\Sigma} \partial_{\delta t} \boldsymbol{\eta}_{h}^{n+1} \cdot \boldsymbol{w}_{h} \\
=\gamma \frac{\mu}{h} \int_{\Sigma} \boldsymbol{u}_{h}^{*} \cdot \boldsymbol{w}_{h}-\int_{\Sigma} \boldsymbol{\sigma}\left(\boldsymbol{u}_{h}^{*}, p_{h}^{*}\right) \boldsymbol{n} \cdot \boldsymbol{w}_{h}
\end{aligned}
$$

for all $\left(\boldsymbol{w}_{h}, \dot{\boldsymbol{w}}_{h}\right) \in X_{h} \times X_{h}$.

- Fluid sub-problem: Given $\left(\boldsymbol{u}_{h}^{*}, p_{h}^{*}\right) \in W_{h} \times Q_{h}$ and $\partial_{\delta t} \boldsymbol{\eta}_{h}^{n+1} \in X_{h}$, find $\left(\boldsymbol{u}_{h}^{n+1}, p_{h}^{n+1}\right) \in W_{h} \times Q_{h}$, with $\boldsymbol{u}_{h}^{n+1}=\overline{\boldsymbol{u}}_{h}\left(t_{n+1}\right)$ on $\Gamma^{\text {in }}$, such that

$$
\begin{array}{r}
A_{\mathrm{f}, \delta t}\left(\left(\boldsymbol{u}_{h}^{n+1}, p_{h}^{+1}\right),\left(\boldsymbol{v}_{h}, q_{h}\right)\right)-\int_{\Sigma} \boldsymbol{\sigma}\left(\boldsymbol{u}_{h}^{*}, p_{h}^{*}\right) \boldsymbol{n} \cdot \boldsymbol{v}_{h}-\int_{\Sigma} \boldsymbol{u}_{h}^{n+1} \cdot \boldsymbol{\sigma}\left(\alpha \boldsymbol{v}_{h},-q_{h}\right) \boldsymbol{n} \\
+\gamma \frac{\mu}{h} \int_{\Sigma} \boldsymbol{u}_{h}^{n+1} \cdot \boldsymbol{v}_{h}=-\int_{\Sigma} \partial_{\partial t} \boldsymbol{\eta}_{h}^{n+1} \cdot \boldsymbol{\sigma}\left(\alpha \boldsymbol{v}_{h},-q_{h}\right) \boldsymbol{n}+\gamma \frac{\mu}{h} \int_{\Sigma} \partial_{\partial t} \boldsymbol{\eta}_{h}^{n+1} \cdot \boldsymbol{v}_{h} \\
+\int_{\Gamma \text { out }} \boldsymbol{g}\left(t_{n+1}\right) \cdot \boldsymbol{v}_{h}, \quad(15)
\end{array}
$$

for all $\left(\boldsymbol{v}_{h}, q_{h}\right) \in V_{h} \times Q_{h}$. 
If $\boldsymbol{u}_{h}^{*} \stackrel{\text { def }}{=} \boldsymbol{u}_{h}^{n+1}$ and $p_{h}^{*} \stackrel{\text { def }}{=} p_{h}^{n+1}$ for $0 \leq n \leq N-1$, the scheme 14 -15 corresponds to an implicit coupling scheme. On the other hand, if $\boldsymbol{u}_{h}^{*} \stackrel{\text { def }}{=} \boldsymbol{u}_{h}^{n}$ and $p_{h}^{*} \stackrel{\text { def }}{=} p_{h}^{n}$ for $0 \leq n \leq N-1$, the scheme is (fully) explicit or loosely coupled.

In the next two paragraphs, we will discuss the stability properties of the numerical schemes resulting from these choices of $\boldsymbol{u}_{h}^{*}$ and $p_{h}^{*}$. In Section $\$ 5$ we will show that the explicit coupling scheme can be stabilized by adding, to the fluid sub-problem, a suitable interface time-penalization term acting on the pressure.

\subsection{Implicit coupling}

For $\boldsymbol{u}_{h}^{*}=\boldsymbol{u}_{h}^{n+1}$ and $p_{h}^{*}=p_{h}^{n+1}, 0 \leq n \leq N-1$, the scheme (14)-(15) corresponds to an implicit coupling scheme. In other words, at each time level, $n$, the subproblems $(14)$ and $(15)$ are fully coupled.

By adding (14) and (15), the implicit scheme can be reformulated (monolithically) as follows: Find $\left(\boldsymbol{u}_{h}^{n+1}, p_{h}^{n+1}, \boldsymbol{\eta}_{h}^{n+1}, \dot{\boldsymbol{\eta}}_{h}^{n+1}\right) \in W_{h} \times Q_{h} \times X_{h} \times X_{h}$, with $\boldsymbol{u}_{h}^{n+1}=\overline{\boldsymbol{u}}_{h}\left(t_{m+1}\right)$ on $\Gamma^{\text {in }}$, such that

$$
\begin{aligned}
& A_{\mathrm{f}, \delta t}\left(\left(\boldsymbol{u}_{h}^{n+1}, p_{h}^{n+1}\right),\left(\boldsymbol{v}_{h}, q_{h}\right)\right)+A_{\mathrm{s}, \delta t}\left(\left(\boldsymbol{\eta}_{h}^{n+1}, \dot{\boldsymbol{\eta}}_{h}^{n+1}\right),\left(\boldsymbol{w}_{h}, \dot{\boldsymbol{w}}_{h}\right)\right) \\
& -\int_{\Sigma} \boldsymbol{\sigma}\left(\boldsymbol{u}_{h}^{n+1}, p_{h}^{n+1}\right) \boldsymbol{n} \cdot\left(\boldsymbol{v}_{h}-\boldsymbol{w}_{h}\right)-\int_{\Sigma}\left(\boldsymbol{u}_{h}^{n+1}-\partial_{\delta t} \boldsymbol{\eta}_{h}^{n+1}\right) \cdot \boldsymbol{\sigma}\left(\alpha \boldsymbol{v}_{h},-q_{h}\right) \boldsymbol{n} \\
& +\gamma \frac{\mu}{h} \int_{\Sigma}\left(\boldsymbol{u}_{h}^{n+1}-\partial_{t} \boldsymbol{\eta}_{h}^{n+1}\right) \cdot\left(\boldsymbol{v}_{h}-\boldsymbol{w}_{h}\right)=\int_{\Gamma \text { out }} \boldsymbol{g}\left(t_{n+1}\right) \cdot \boldsymbol{v}_{h},
\end{aligned}
$$

for all $\left(\boldsymbol{v}_{h}, q_{h}, \boldsymbol{w}_{h}, \dot{\boldsymbol{w}}_{h}\right) \in V_{h} \times Q_{h} \times X_{h} \times X_{h}$, which is a fully discrete counterpart of (4).

In order to simplify the presentation, we introduce the following notations: $\boldsymbol{U}_{h}^{n+1} \stackrel{\text { def }}{=}\left(\boldsymbol{u}_{h}^{n+1}, p_{h}^{n+1}\right)$ and $\boldsymbol{V}_{h} \stackrel{\text { def }}{=}\left(\boldsymbol{v}_{h}, q_{h}\right)$ stand for the fluid state and test functions, $\boldsymbol{\Theta}_{h}^{n+1} \stackrel{\text { def }}{=}\left(\boldsymbol{\eta}_{h}^{n+1}, \dot{\boldsymbol{\eta}}_{h}^{n+1}\right)$ and $\boldsymbol{W}_{h} \stackrel{\text { def }}{=}\left(\boldsymbol{w}_{h}, \dot{\boldsymbol{w}}_{h}\right)$ for the solid state and test functions and

$$
\begin{aligned}
A_{\mathrm{I}}\left[\left(\boldsymbol{U}_{h}^{n+1}, \Theta_{h}^{n+1}\right),\left(\boldsymbol{V}_{h}, \boldsymbol{W}_{h}\right)\right] \stackrel{\text { def }}{=} & A_{\mathrm{f}, \delta t}\left(\left(\boldsymbol{u}_{h}^{n+1}, p_{h}^{n+1}\right),\left(\boldsymbol{v}_{h}, q_{h}\right)\right) \\
& +A_{\mathrm{s}, \delta t}\left(\left(\boldsymbol{\eta}_{h}^{n+1}, \dot{\boldsymbol{\eta}}_{h}^{n+1}\right),\left(\boldsymbol{w}_{h}, \dot{\boldsymbol{w}}_{h}\right)\right) \\
& -\int_{\Sigma} \boldsymbol{\sigma}\left(\boldsymbol{u}_{h}^{n+1}, p_{h}^{n+1}\right) \boldsymbol{n} \cdot\left(\boldsymbol{v}_{h}-\boldsymbol{w}_{h}\right) \\
& -\int_{\Sigma}\left(\boldsymbol{u}_{h}^{n+1}-\partial_{\delta t} \boldsymbol{\eta}_{h}^{n+1}\right) \cdot \boldsymbol{\sigma}\left(\alpha \boldsymbol{v}_{h},-q_{h}\right) \boldsymbol{n} \\
& +\gamma \frac{\mu}{h} \int_{\Sigma}\left(\boldsymbol{u}_{h}^{n+1}-\partial_{t} \boldsymbol{\eta}_{h}^{n+1}\right) \cdot\left(\boldsymbol{v}_{h}-\boldsymbol{w}_{h}\right),
\end{aligned}
$$

for the implicit coupling bilinear form. As a result, (16) simply reduces to

$$
A_{\mathrm{I}}\left[\left(\boldsymbol{U}_{h}^{n+1}, \Theta_{h}^{n+1}\right),\left(\boldsymbol{V}_{h}, \boldsymbol{W}_{h}\right)\right]=\int_{\Gamma \text { out }} \boldsymbol{g}\left(t_{n+1}\right) \cdot \boldsymbol{v}_{h},
$$

for all $\left(\boldsymbol{V}_{h}, \boldsymbol{W}_{h}\right) \in\left(V_{h} \times Q_{h}\right) \times X_{h}^{2}$. 


\subsubsection{Stability analysis - implicit coupling}

As one could expect, implicit coupling is unconditionally stable in the energy norm. Let $E^{n}$ denote the total discrete energy of the system at the time level $n$, defined by

$$
E^{n} \stackrel{\text { def }}{=} E_{\mathrm{f}}^{n}+E_{\mathrm{s}}^{n}+\frac{3 \mu}{4} \delta t \sum_{m=0}^{n-1}\left\|\boldsymbol{\epsilon}\left(\boldsymbol{u}_{h}^{m+1}\right)\right\|_{0, \Omega_{\mathrm{f}}}^{2}+\frac{3}{4} \frac{\gamma \mu}{h} \delta t \sum_{m=0}^{n-1}\left\|\boldsymbol{u}_{h}^{m+1}-\partial_{t} \boldsymbol{\eta}_{h}^{m+1}\right\|_{0, \Sigma}^{2},
$$

with

$$
E_{\mathrm{f}}^{n} \stackrel{\text { def }}{=} \frac{\rho_{\mathrm{f}}}{2}\left\|\boldsymbol{u}_{h}^{n}\right\|_{0, \Omega_{\mathrm{f}}}^{2}, \quad E_{\mathrm{s}}^{n} \stackrel{\text { def }}{=} \frac{\rho_{\mathrm{s}}}{2}\left\|\dot{\boldsymbol{\eta}}_{h}^{n}\right\|_{0, \Omega_{\mathrm{s}}}^{2}+\frac{1}{2} a_{\mathrm{s}}\left(\boldsymbol{\eta}_{h}^{n}, \boldsymbol{\eta}_{h}^{n}\right) .
$$

The next Lemma states the unconditional stability of the implicit coupling scheme, 14)-15 with $\boldsymbol{u}_{h}^{*} \stackrel{\text { def }}{=} \boldsymbol{u}_{h}^{n+1}$ and $p_{h}^{*} \stackrel{\text { def }}{=} p_{h}^{n+1}$.

Lemma 4.1 Assume that the fluid-structure system is isolated, i.e. $\overline{\boldsymbol{u}}=\mathbf{0}$ and $\boldsymbol{g}=\mathbf{0}$. Let $\left\{\left(\boldsymbol{u}_{h}^{n+1}, p_{h}^{n+1}, \boldsymbol{\eta}_{h}^{n+1}, \dot{\boldsymbol{\eta}}_{h}^{n+1}\right)\right\}_{0 \leq n \leq N-1}$ the solution of 14 - 15 with $\boldsymbol{u}_{h}^{*} \stackrel{\text { def }}{=} \boldsymbol{u}_{h}^{n+1}$ and $p_{h}^{*} \stackrel{\text { def }}{=} p_{h}^{n+1}$. Then, under the condition (6), the following energy estimate holds,

$$
E^{n} \leq E^{0}
$$

with $1 \leq n \leq N-1$. In particular, for $\alpha=-1$ this estimate holds true for all values of the Nitsche's penalty parameter $\gamma>0$.

Proof. The proof follows, with minor modifications, the proof of Lemma 3.3 for the space semi-discrete case. Indeed, by testing 18 with

$$
\left(\boldsymbol{v}_{h}, q_{h}, \boldsymbol{w}_{h}, \dot{\boldsymbol{w}}_{h}\right)=\left(\boldsymbol{u}_{h}^{n+1}, p_{h}^{n+1}, \partial_{\delta t} \boldsymbol{\eta}_{h}^{n+1}, \partial_{\delta t} \dot{\boldsymbol{\eta}}_{h}^{n+1}\right),
$$

using (9), replacing index $n$ by $m$ and summing over $0 \leq m \leq n-1$, using condition $\sqrt{60}$ and the definition $\sqrt{19}$, we get

$$
E^{n} \leq \frac{\rho_{\mathrm{f}}}{2}\left\|\boldsymbol{u}_{h}^{0}\right\|_{0, \Omega_{\mathrm{f}}}^{2}+\frac{\rho_{\mathrm{s}}}{2}\left\|\dot{\boldsymbol{\eta}}_{h}^{0}\right\|_{0, \Omega_{\mathrm{s}}}^{2}+\frac{1}{2} a_{\mathrm{s}}\left(\boldsymbol{\eta}_{h}^{0}, \boldsymbol{\eta}_{h}^{0}\right)
$$

which completes the proof. $\square$

Despite the outstanding stability properties provided by the previous Lemma, implicit coupling has the major disadvantage of being too CPU-time consuming. Indeed, at each time-level $n$, it involves the solution of the fully coupled system (18), which can be solved monolithically, by treating (18) as an individual problem, or in a partitioned fashion, by sub-iterating (until convergence!) between the fluid and solid subproblems (14)- 15 .

\section{$4.2 \quad$ Explicit coupling}

For $\boldsymbol{u}_{h}^{*}=\boldsymbol{u}_{h}^{n}$ and $p_{h}^{*}=p_{h}^{n}, 0 \leq n \leq N-1$, the scheme 14)-15 is explicit (or loosely coupled). In such a procedure, one can advance in time by solving first for the solid in (14), since $\left(\boldsymbol{u}_{h}^{n}, p_{h}^{n}\right)$ is known from the previous time level, and then for the fluid in (15), since $\partial_{\delta t} \boldsymbol{\eta}_{h}^{n+1}$ is provided by the previous solid step. This simple splitting procedure, for solving fluid-structure interaction problems, is also known in the literature as conventional serial staggered (CSS) scheme (see e.g. [31, 11]). 
Clearly, explicit coupling is very appealing in terms of efficiency: it only involves one (or a few) solution of the fluid and solid sub-problems per time step. However, this fluid-solid splitting can drastically compromise the numerical stability of the scheme, as pointed out in [26, 24, 30, 7, 16], when dealing with incompressible fluids.

To illustrate this issue, in the case of the partitioned Nitsche fluid-structure formulation (14)- 15 , we reformulate the explicit coupling scheme in a monolithic fashion, as we did for the implicit scheme in (18), by adding the expressions (14) and (15). This yields the global problem: Find $\left(\boldsymbol{u}_{h}^{n+1}, p_{h}^{n+1}, \boldsymbol{\eta}_{h}^{n+1}, \dot{\boldsymbol{\eta}}_{h}^{n+1}\right) \in$ $W_{h} \times Q_{h} \times X_{h} \times X_{h}$, with $\boldsymbol{u}_{h}^{n+1}=\overline{\boldsymbol{u}}_{h}\left(t_{n+1}\right)$ on $\Gamma^{\text {in }}$, such that

$$
\begin{aligned}
& A_{\mathrm{f}, \delta t}\left(\left(\boldsymbol{u}_{h}^{n+1}, p_{h}^{n+1}\right),\left(\boldsymbol{v}_{h}, q_{h}\right)\right)+A_{\mathrm{s}, \delta t}\left(\left(\boldsymbol{\eta}_{h}^{n+1}, \dot{\boldsymbol{\eta}}_{h}^{n+1}\right),\left(\boldsymbol{w}_{h}, \dot{\boldsymbol{w}}_{h}\right)\right)-\int_{\Sigma} \boldsymbol{\sigma}\left(\boldsymbol{u}_{h}^{n}, p_{h}^{n}\right) \boldsymbol{n} \cdot \boldsymbol{v}_{h} \\
& +\int_{\Sigma} \boldsymbol{\sigma}\left(\boldsymbol{u}_{h}^{n}, p_{h}^{n}\right) \boldsymbol{n} \cdot \boldsymbol{w}_{h}-\int_{\Sigma}\left(\boldsymbol{u}_{h}^{n+1}-\partial_{\delta t} \boldsymbol{\eta}_{h}^{n+1}\right) \cdot \boldsymbol{\sigma}\left(\alpha \boldsymbol{v}_{h},-q_{h}\right) \boldsymbol{n} \\
& +\gamma \frac{\mu}{h} \int_{\Sigma}\left(\boldsymbol{u}_{h}^{n+1}-\partial_{t} \boldsymbol{\eta}_{h}^{n+1}\right) \cdot \boldsymbol{v}_{h}-\gamma \frac{\mu}{h} \int_{\Sigma}\left(\boldsymbol{u}_{h}^{n}-\partial_{t} \boldsymbol{\eta}_{h}^{n+1}\right) \cdot \boldsymbol{w}_{h}=\int_{\Gamma \text { out }} \boldsymbol{g}\left(t_{n+1}\right) \cdot \boldsymbol{v}_{h}
\end{aligned}
$$

for all $\left(\boldsymbol{v}_{h}, q_{h}, \boldsymbol{w}_{h}, \dot{\boldsymbol{w}}_{h}\right) \in V_{h} \times Q_{h} \times X_{h} \times X_{h}$, which is an explicit coupling, fully discrete, counterpart of (4). Equivalently, in a more compact form,

$$
A_{\mathrm{E}}\left[\left(\boldsymbol{U}_{h}^{n+1}, \Theta_{h}^{n+1}\right),\left(\boldsymbol{V}_{h}, \boldsymbol{W}_{h}\right)\right]=\int_{\Gamma_{\text {out }}} \boldsymbol{g}^{n+1} \cdot \boldsymbol{v}_{h},
$$

for all $\left(\boldsymbol{V}_{h}, \boldsymbol{W}_{h}\right) \in\left(V_{h} \times Q_{h}\right) \times X_{h}^{2}$, where the explicit bilinear form $A_{\mathrm{E}}$ is given by

$$
\begin{aligned}
A_{\mathrm{E}}\left[\left(\boldsymbol{U}_{h}^{n+1}, \boldsymbol{\Theta}_{h}^{n+1}\right),\left(\boldsymbol{V}_{h}, \boldsymbol{W}_{h}\right)\right] \stackrel{\text { def }}{=} & A_{\mathrm{f}, \delta t}\left(\left(\boldsymbol{u}_{h}^{n+1}, p_{h}^{n+1}\right),\left(\boldsymbol{v}_{h}, q_{h}\right)\right) \\
& +A_{\mathrm{s}, \delta t}\left(\left(\boldsymbol{\eta}_{h}^{n+1}, \dot{\boldsymbol{\eta}}_{h}^{n+1}\right),\left(\boldsymbol{w}_{h}, \dot{\boldsymbol{w}}_{h}\right)\right) \\
& -\int_{\Sigma} \boldsymbol{\sigma}\left(\boldsymbol{u}_{h}^{n}, p_{h}^{n}\right) \boldsymbol{n} \cdot\left(\boldsymbol{v}_{h}-\boldsymbol{w}_{h}\right) \\
& -\int_{\Sigma}\left(\boldsymbol{u}_{h}^{n+1}-\partial_{\delta t} \boldsymbol{\eta}_{h}^{n+1}\right) \cdot \boldsymbol{\sigma}\left(\alpha \boldsymbol{v}_{h},-q_{h}\right) \boldsymbol{n} \\
& +\gamma \frac{\mu}{h} \int_{\Sigma}\left(\boldsymbol{u}_{h}^{n+1}-\partial_{\delta t} \boldsymbol{\eta}_{h}^{n+1}\right) \cdot \boldsymbol{v}_{h} \\
& -\gamma \frac{\mu}{h} \int_{\Sigma}\left(\boldsymbol{u}_{h}^{n}-\partial_{\delta t} \boldsymbol{\eta}_{h}^{n+1}\right) \cdot \boldsymbol{w}_{h} .
\end{aligned}
$$

From (17), it then follows that

$$
\begin{aligned}
& A_{\mathrm{E}}\left[\left(\boldsymbol{U}_{h}^{n+1}, \boldsymbol{\Theta}_{h}^{n+1}\right),\left(\boldsymbol{V}_{h}, \boldsymbol{W}_{h}\right)\right]=A_{\mathrm{I}}\left[\left(\boldsymbol{U}_{h}^{n+1}, \boldsymbol{\Theta}_{h}^{n+1}\right),\left(\boldsymbol{V}_{h}, \boldsymbol{W}_{h}\right)\right] \\
+ & \gamma \frac{\mu}{h} \int_{\Sigma}\left(\boldsymbol{u}_{h}^{n+1}-\boldsymbol{u}_{h}^{n}\right) \cdot \boldsymbol{w}_{h}+\int_{\Sigma}\left(\boldsymbol{\sigma}\left(\boldsymbol{u}_{h}^{n+1}, p_{h}^{n+1}\right) \boldsymbol{n}-\boldsymbol{\sigma}\left(\boldsymbol{u}_{h}^{n}, p_{h}^{n}\right) \boldsymbol{n}\right) \cdot\left(\boldsymbol{v}_{h}-\boldsymbol{w}_{h}\right) .
\end{aligned}
$$

Therefore, the explicit coupling scheme can be thought of as an interface perturbation of the implicit coupling scheme. This perturbation consists of two terms, related to the time variations of the fluid velocity and fluid stresses at the interface $\Sigma$. In the next paragraph we will see that the dissipative character 
of the Nitsche's coupling is able to control the first perturbation and the viscous contribution of the second, under suitable conditions on $\gamma, h$ and $\delta t$ (see Remarks 5.5 and 5.6). The pressure contribution of the second perturbation, on the other hand, needs a special treatment, which will be the topic of Section $\$ 5$.

\subsubsection{Stability analysis - explicit coupling}

Let us stress that the purpose of this paragraph is simply to illustrate (not to prove) the instability of the explicit coupling scheme $(14)-(15)$. The discussion below will motivate the introduction of a new (stabilized) explicit coupling scheme in Section $\$ 5$.

Lemma 4.2 Assume that the fluid-structure system is isolated, i.e. $\overline{\boldsymbol{u}}=\mathbf{0}$ and $\boldsymbol{g}=\mathbf{0}$. Let $\left\{\left(\boldsymbol{u}_{h}^{n+1}, p_{h}^{n+1}, \boldsymbol{\eta}_{h}^{n+1}, \dot{\boldsymbol{\eta}}_{h}^{n+1}\right)\right\}_{0 \leq n \leq N-1}$ the solution of 14 - 15 with $\boldsymbol{u}_{h}^{*} \stackrel{\text { def }}{=} \boldsymbol{u}_{h}^{n}$ and $p_{h}^{*} \stackrel{\text { def }}{=} p_{h}^{n}$. Then, under the conditions

$$
\begin{aligned}
\gamma & \geq 256 C_{\mathrm{TI}}, \\
\gamma \delta t & \leq C_{\Sigma} h,
\end{aligned}
$$

with $C_{\Sigma}>0$ a given constant, the following estimate holds,

$$
\begin{aligned}
E^{n}+3 \delta t \frac{\gamma \mu}{h}\left\|\boldsymbol{u}_{h}^{n}\right\|_{0, \Sigma}^{2} \leq 6 E^{0}+3 C_{\Sigma} \mu\left\|\boldsymbol{u}_{h}^{0}\right\|_{0, \Sigma}^{2} & +\frac{3 \mu}{4}\left\|\boldsymbol{\epsilon}\left(\boldsymbol{u}_{h}^{0}\right)\right\|_{0, \Omega_{\mathrm{f}}}^{2} \\
& +24 \frac{h}{\gamma \mu} \delta t \sum_{m=0}^{n-1}\left\|p_{h}^{m+1}-p_{h}^{m}\right\|_{0, \Sigma}^{2} .
\end{aligned}
$$

Proof. By testing 21] with

$$
\left(\boldsymbol{v}_{h}, q_{h}, \boldsymbol{w}_{h}, \dot{\boldsymbol{w}}_{h}\right)=\left(\boldsymbol{u}_{h}^{n+1}, p_{h}^{n+1}, \partial_{\delta t} \boldsymbol{\eta}_{h}^{n+1}, \partial_{\delta t} \dot{\boldsymbol{\eta}}_{h}^{n+1}\right),
$$

using (22), multiplying by $\delta t$, replacing index $n$ by $m$ and summing over $0 \leq$ $m \leq n-1$ and using the stability analysis of the implicit scheme (note that condition (23) implies (6), we have

$$
\begin{aligned}
& E^{n} \leq E^{0}-\underbrace{\frac{\gamma \mu}{h} \delta t \sum_{m=0}^{n-1} \int_{\Sigma}\left(\boldsymbol{u}_{h}^{m+1}-\boldsymbol{u}_{h}^{m}\right) \cdot \partial_{\delta t} \boldsymbol{\eta}_{h}^{m+1}}_{T_{1}} \\
& -\delta t \sum_{m=0}^{n-1} \int_{\Sigma}\left(\boldsymbol{\sigma}\left(\boldsymbol{u}_{h}^{m+1}, p_{h}^{m+1}\right) \boldsymbol{n}-\boldsymbol{\sigma}\left(\boldsymbol{u}_{h}^{m}, p_{h}^{m}\right) \boldsymbol{n}\right) \cdot\left(\boldsymbol{u}_{h}^{m+1}-\partial_{\delta t} \boldsymbol{\eta}_{h}^{m+1}\right) \\
& =E^{0}-T_{1}-\underbrace{\delta t \sum_{m=0}^{n-1} \int_{\Sigma} 2 \mu\left(\boldsymbol{\epsilon}\left(\boldsymbol{u}_{h}^{m+1}\right) \boldsymbol{n}-\boldsymbol{\epsilon}\left(\boldsymbol{u}_{h}^{m}\right) \boldsymbol{n}\right) \cdot\left(\boldsymbol{u}_{h}^{m+1}-\partial_{\delta t} \boldsymbol{\eta}_{h}^{m+1}\right)}_{T_{2}} \\
& +\underbrace{\delta t \sum_{m=0}^{n-1} \int_{\Sigma}\left(p_{h}^{m+1}-p_{h}^{m}\right)\left(\boldsymbol{u}_{h}^{m+1}-\partial_{\delta t} \boldsymbol{\eta}_{h}^{m+1}\right) \cdot \boldsymbol{n}}_{T_{3}} .
\end{aligned}
$$


As mentioned above, the term $T_{1}$ involving the fluid velocity fluctuations at the interface can be handled using the Nitsche's penalty coupling term. Indeed, we have

$$
\begin{aligned}
T_{1} & =\frac{\gamma \mu}{h} \delta t \sum_{m=0}^{n-1} \int_{\Sigma}\left(\boldsymbol{u}_{h}^{m+1}-\boldsymbol{u}_{h}^{m}\right) \cdot\left(\boldsymbol{u}_{h}^{m+1}+\partial_{\delta t} \boldsymbol{\eta}_{h}^{m+1}-\boldsymbol{u}_{h}^{m+1}\right) \\
& =\frac{\gamma \mu}{h} \delta t \sum_{m=0}^{n-1}\left[\int_{\Sigma}\left(\boldsymbol{u}_{h}^{m+1}-\boldsymbol{u}_{h}^{m}\right) \cdot \boldsymbol{u}_{h}^{m+1}-\int_{\Sigma}\left(\boldsymbol{u}_{h}^{m+1}-\boldsymbol{u}_{h}^{m}\right) \cdot\left(\boldsymbol{u}_{h}^{m+1}-\partial_{\delta t} \boldsymbol{\eta}_{h}^{m+1}\right)\right] \\
& \geq \frac{\gamma \mu}{2 h} \delta t \sum_{m=0}^{n-1}\left(\left\|\boldsymbol{u}_{h}^{m+1}\right\|_{0, \Sigma}^{2}-\left\|\boldsymbol{u}_{h}^{m}\right\|_{0, \Sigma}^{2}-\left\|\boldsymbol{u}_{h}^{m+1}-\partial_{\delta t} \boldsymbol{\eta}_{h}^{m+1}\right\|_{0, \Sigma}^{2}\right) .
\end{aligned}
$$

Therefore, using (24) we obtain

$$
T_{1} \geq \delta t \frac{\gamma \mu}{2 h}\left\|\boldsymbol{u}_{h}^{n}\right\|_{0, \Sigma}^{2}-\frac{C_{\Sigma}}{2} \mu\left\|\boldsymbol{u}_{h}^{0}\right\|_{0, \Sigma}^{2}-\frac{\gamma \mu}{2 h} \delta t \sum_{m=0}^{n-1}\left\|\boldsymbol{u}_{h}^{m+1}-\partial_{\delta t} \boldsymbol{\eta}_{h}^{m+1}\right\|_{0, \Sigma}^{2} .
$$

On the other hand, using Young's inequality and the local trace-inverse inequality (5), we have (for $\epsilon_{1}>0$ )

$$
\begin{aligned}
T_{2} \geq & -\frac{\mu}{\epsilon_{1} \gamma} \delta t \sum_{m=0}^{n-1} h\left\|\boldsymbol{\epsilon}\left(\boldsymbol{u}_{h}^{m+1}\right) \boldsymbol{n}-\boldsymbol{\epsilon}\left(\boldsymbol{u}_{h}^{m}\right) \boldsymbol{n}\right\|_{0, \Sigma}^{2}-\frac{\epsilon_{1} \gamma \mu}{h} \delta t \sum_{m=0}^{n-1}\left\|\boldsymbol{u}_{h}^{m+1}-\partial_{\delta t} \boldsymbol{\eta}_{h}^{m+1}\right\|_{0, \Sigma}^{2} \\
\geq & -\frac{\mu C_{\mathrm{TI}}}{\epsilon_{1} \gamma} \delta t \sum_{m=0}^{n-1}\left\|\boldsymbol{\epsilon}\left(\boldsymbol{u}_{h}^{m+1}\right)-\boldsymbol{\epsilon}\left(\boldsymbol{u}_{h}^{m}\right)\right\|_{0, \Omega_{\mathrm{f}}}^{2}-\frac{\epsilon_{1} \gamma \mu}{h} \delta t \sum_{m=0}^{n-1}\left\|\boldsymbol{u}_{h}^{m+1}-\partial_{\delta t} \boldsymbol{\eta}_{h}^{m+1}\right\|_{0, \Sigma}^{2} \\
\geq & -2 \delta t \frac{\mu C_{\mathrm{TI}}}{\epsilon_{1} \gamma}\left\|\boldsymbol{\epsilon}\left(\boldsymbol{u}_{h}^{0}\right)\right\|_{0, \Omega_{\mathrm{f}}}^{2}-4 \frac{\mu C_{\mathrm{TI}}}{\epsilon_{1} \gamma} \delta t \sum_{m=0}^{n-1}\left\|\boldsymbol{\epsilon}\left(\boldsymbol{u}_{h}^{m+1}\right)\right\|_{0, \Omega_{\mathrm{f}}}^{2} \\
& -\frac{\epsilon_{1} \gamma \mu}{h} \delta t \sum_{m=0}^{n-1}\left\|\boldsymbol{u}_{h}^{m+1}-\partial_{\delta t} \boldsymbol{\eta}_{h}^{m+1}\right\|_{0, \Sigma}^{2} .
\end{aligned}
$$

Finally, using Young's inequality, for the last term we have $\left(\epsilon_{2}>0\right)$

$$
T_{3} \leq \frac{1}{4 \epsilon_{2}} \frac{h}{\gamma \mu} \delta t \sum_{m=0}^{n-1}\left\|p_{h}^{m+1}-p_{h}^{m}\right\|_{0, \Sigma}^{2}+\frac{\epsilon_{2} \gamma \mu}{h} \delta t \sum_{m=0}^{n-1}\left\|\boldsymbol{u}_{h}^{m+1}-\partial_{\delta t} \boldsymbol{\eta}_{h}^{m+1}\right\|_{0, \Sigma}^{2}
$$

Therefore, by inserting the estimates $(27)-(29)$ in $(26)$, we obtain

$$
\begin{aligned}
E_{\mathrm{f}}^{n} & +E_{\mathrm{s}}^{n}+\mu\left(\frac{3}{4}-4 \frac{C_{\mathrm{TI}}}{\epsilon_{1} \gamma}\right) \delta t \sum_{m=0}^{n-1}\left\|\boldsymbol{\epsilon}\left(\boldsymbol{u}_{h}^{m+1}\right)\right\|_{0, \Omega_{\mathrm{f}}}^{2} \\
& +\left(\frac{3}{4}-\frac{1}{2}-\epsilon_{1}-\epsilon_{2}\right) \frac{\gamma \mu}{h} \delta t \sum_{m=0}^{n-1}\left\|\boldsymbol{u}_{h}^{m+1}-\partial_{t} \boldsymbol{\eta}_{h}^{m+1}\right\|_{0, \Sigma}^{2}+\delta t \frac{\gamma \mu}{2 h}\left\|\boldsymbol{u}_{h}^{n}\right\|_{0, \Sigma}^{2} \\
\leq & E^{0}+\frac{C_{\Sigma}}{2} \mu\left\|\boldsymbol{u}_{h}^{0}\right\|_{0, \Sigma}^{2}+2 \delta t \frac{\mu C_{\mathrm{TI}}}{\epsilon_{1} \gamma}\left\|\boldsymbol{\epsilon}\left(\boldsymbol{u}_{h}^{0}\right)\right\|_{0, \Omega_{\mathrm{f}}}^{2}+\frac{1}{4 \epsilon_{2}} \frac{h}{\gamma \mu} \delta t \sum_{m=0}^{n-1}\left\|p_{h}^{m+1}-p_{h}^{m}\right\|_{0, \Sigma}^{2} .
\end{aligned}
$$


Thus, by taking $\epsilon_{1}=\epsilon_{2}=1 / 16$ and under condition 23 , we get

$$
\begin{gathered}
E_{\mathrm{f}}^{n}+E_{\mathrm{s}}^{n}+\frac{\mu}{2} \delta t \sum_{m=0}^{n-1}\left\|\boldsymbol{\epsilon}\left(\boldsymbol{u}_{h}^{m+1}\right)\right\|_{0, \Omega_{\mathrm{f}}}^{2}+\frac{1}{8} \frac{\gamma \mu}{h} \delta t \sum_{m=0}^{n-1}\left\|\boldsymbol{u}_{h}^{m+1}-\partial_{t} \boldsymbol{\eta}_{h}^{m+1}\right\|_{0, \Sigma}^{2} \\
+\delta t \frac{\gamma \mu}{2 h}\left\|\boldsymbol{u}_{h}^{n}\right\|_{0, \Sigma}^{2} \leq E^{0}+\frac{C_{\Sigma}}{2} \mu\left\|\boldsymbol{u}_{h}^{0}\right\|_{0, \Sigma}^{2}+\delta t \frac{\mu}{8}\left\|\boldsymbol{\epsilon}\left(\boldsymbol{u}_{h}^{0}\right)\right\|_{0, \Omega_{\mathrm{f}}}^{2}+4 \frac{h}{\gamma \mu} \delta t \sum_{m=0}^{n-1}\left\|p_{h}^{m+1}-p_{h}^{m}\right\|_{0, \Sigma}^{2} \cdot
\end{gathered}
$$

which completes the proof. $\square$

The above Lemma shows that, under the CFL-like condition (24), the Nitsche interface penalty and the viscous dissipation in $E^{n}$ control the first perturbation term in 22 and the interface viscous stress contribution of the the second perturbation term in 22 . Unfortunately, the remaining pressure fluctuations contribution in 22 can not be directly controlled by the discrete energy of the system $E^{n}$. Actually, the energy estimate does not provide a control of the pressure on the interface. Somehow this illustrates the infamous numerical instability featured by the explicit coupling scheme, when dealing with incompressible fluids.

We refer to [7] for a rigorous explanation of this issue in a simplified time semi-discrete framework, relating the instability of the scheme to the addedmass effect (fluid-solid density ratio and length of the domain), irrespectively of the discretization parameters. A further analysis, considering different time discretization schemes, has been recently reported in [16. In particular, the authors conclude that no sequentially staggered scheme can be constructed which would be unconditionally stable with respect to to the fluid-solid density ratio.

Based on the above analysis, in the next section we propose a new explicit coupling scheme which, under condition (24), is unconditionally stable with respect to the added-mass effect.

Remark 4.3 In the explicit coupling version of (14)-(15), the consistency term $-\int_{\Sigma} \boldsymbol{\sigma}\left(\boldsymbol{u}_{h}, p_{h}\right) \boldsymbol{n} \cdot \boldsymbol{v}_{h}$ of the fluid sub-problem could also be evaluated at time level $n+1$, as originally proposed in [4]. However, in this case the viscous part of the new interface stress perturbation term in 22 can not be controlled by the viscous fluid dissipation and the Nitsche's penalty term. This shows that the explicit treatment of this term becomes natural within a explicit coupling framework (see also Remark 5.1 in the next section).

\section{$5 \quad$ Stabilized explicit coupling}

In order to control the spurious oscillations of the fluid pressure at the interface, arising in the energy estimate (25), we add to the fluid sub-problem 15 the following weakly consistent penalty term:

$$
S\left(p_{h}^{n+1}, q_{h}\right) \stackrel{\text { def }}{=} \frac{\gamma_{0} h}{\gamma \mu} \int_{\Sigma}\left(p_{h}^{n+1}-p_{h}^{n}\right) q_{h}
$$

with $\gamma_{0}>0$ a (dimensionless) parameter to be chosen sufficiently large (see Theorem 5.2. Clearly, this corresponds to penalize the fluid pressure fluctuations on the fluid-structure interface $\Sigma$.

As a result, our new explicit coupling scheme is given by: 
Stabilized explicit coupling.

- Time loop: For $0 \leq n \leq N-1$ solve:

1. Solid sub-problem: Given $\left(\boldsymbol{u}_{h}^{n}, p_{h}^{n}\right) \in W_{h} \times Q_{h}$, find $\left(\boldsymbol{\eta}_{h}^{n+1}, \dot{\boldsymbol{\eta}}_{h}^{n+1}\right) \in$ $X_{h} \times X_{h}$ such that

$$
\begin{aligned}
A_{\mathrm{s}, \delta t}\left(\left(\boldsymbol{\eta}_{h}^{n+1}, \dot{\boldsymbol{\eta}}_{h}^{n+1}\right),\right. & \left.\left(\boldsymbol{w}_{h}, \dot{\boldsymbol{w}}_{h}\right)\right)+\gamma \frac{\mu}{h} \int_{\Sigma} \partial_{\delta t} \boldsymbol{\eta}_{h}^{n+1} \cdot \boldsymbol{w}_{h} \\
& =\gamma \frac{\mu}{h} \int_{\Sigma} \boldsymbol{u}_{h}^{n} \cdot \boldsymbol{w}_{h}-\int_{\Sigma} \boldsymbol{\sigma}\left(\boldsymbol{u}_{h}^{n}, p_{h}^{n}\right) \boldsymbol{n} \cdot \boldsymbol{w}_{h}
\end{aligned}
$$

for all $\left(\boldsymbol{w}_{h}, \dot{\boldsymbol{w}}_{h}\right) \in X_{h} \times X_{h}$.

2. Fluid sub-problem: Given $\partial_{\delta t} \boldsymbol{\eta}_{h}^{n+1} \in X_{h}$, find $\left(\boldsymbol{u}_{h}^{n+1}, p_{h}^{n+1}\right) \in$ $W_{h} \times Q_{h}$, with $\boldsymbol{u}_{h}^{m+1}=\overline{\boldsymbol{u}}_{h}\left(t_{m+1}\right)$ on $\Gamma^{\text {in }}$, such that

$$
\begin{gathered}
A_{\mathrm{f}, \delta t}\left(\left(\boldsymbol{u}_{h}^{n+1}, p_{h}^{n+1}\right),\left(\boldsymbol{v}_{h}, q_{h}\right)\right)-\int_{\Sigma} \boldsymbol{u}_{h}^{n+1} \cdot \boldsymbol{\sigma}\left(\alpha \boldsymbol{v}_{h},-q_{h}\right) \boldsymbol{n}+\gamma \frac{\mu}{h} \int_{\Sigma} \boldsymbol{u}_{h}^{n+1} \cdot \boldsymbol{v}_{h} \\
+S\left(p_{h}^{n+1}, q_{h}\right)=\int_{\Sigma} \boldsymbol{\sigma}\left(\boldsymbol{u}_{h}^{n}, p_{h}^{n}\right) \boldsymbol{n} \cdot \boldsymbol{v}_{h}-\int_{\Sigma} \partial_{\delta t} \boldsymbol{\eta}_{h}^{n+1} \cdot \boldsymbol{\sigma}\left(\alpha \boldsymbol{v}_{h},-q_{h}\right) \boldsymbol{n} \\
+\gamma \frac{\mu}{h} \int_{\Sigma} \partial_{\delta t} \boldsymbol{\eta}_{h}^{n+1} \cdot \boldsymbol{v}_{h}+\int_{\Gamma \text { out }} \boldsymbol{g}\left(t_{n+1}\right) \cdot \boldsymbol{v}_{h}, \quad \text { (32) }
\end{gathered}
$$

for all $\left(\boldsymbol{v}_{h}, q_{h}\right) \in V_{h} \times Q_{h}$. 
Remark 5.1 The time penalty (30) differs from the original stabilization term proposed by the authors in [4]. On one hand, expression (30) only involves the time pressure variations whereas in [4] also the viscous contribution is included. On the other hand, the scaling factor in (30) makes $\gamma_{0}$ a dimensionless parameter, which is convenient from a practical point of view, whereas in [4] $\gamma_{0}$ has the dimensions of a length. These two features of (30) are a directly related to the explicit treatment of the consistency term $-\int_{\Sigma} \boldsymbol{\sigma}\left(\boldsymbol{u}_{h}, p_{h}\right) \boldsymbol{n} \cdot \boldsymbol{v}_{h}$ in $(32)$, as noticed in Remark 4.3 .

For the stability analysis below, we reformulate this new explicit coupling scheme in a monolithic fashion, by adding the expressions (31) and (32). This yields the problem: Find $\left(\boldsymbol{u}_{h}^{n+1}, p_{h}^{n+1}, \boldsymbol{\eta}_{h}^{n+1}, \dot{\boldsymbol{\eta}}_{h}^{n+1}\right) \in W_{h} \times Q_{h} \times X_{h} \times X_{h}$, with $\boldsymbol{u}_{h}^{n+1}=\overline{\boldsymbol{u}}_{h}\left(t_{n+1}\right)$ on $\Gamma^{\text {in }}$, such that

$$
\begin{aligned}
& A_{\mathrm{f}, \delta t}\left(\left(\boldsymbol{u}_{h}^{n+1}, p_{h}^{n+1}\right),\left(\boldsymbol{v}_{h}, q_{h}\right)\right)+A_{\mathrm{s}, \delta t}\left(\left(\boldsymbol{\eta}_{h}^{n+1}, \dot{\boldsymbol{\eta}}_{h}^{n+1}\right),\left(\boldsymbol{w}_{h}, \dot{\boldsymbol{w}}_{h}\right)\right) \\
& -\int_{\Sigma} \boldsymbol{\sigma}\left(\boldsymbol{u}_{h}^{n}, p_{h}^{n}\right) \boldsymbol{n} \cdot\left(\boldsymbol{v}_{h}-\boldsymbol{w}_{h}\right)-\int_{\Sigma}\left(\boldsymbol{u}_{h}^{n+1}-\partial_{\delta t} \boldsymbol{\eta}_{h}^{n+1}\right) \cdot \boldsymbol{\sigma}\left(\alpha \boldsymbol{v}_{h},-q_{h}\right) \boldsymbol{n} \\
& +\gamma \frac{\mu}{h} \int_{\Sigma}\left(\boldsymbol{u}_{h}^{n+1}-\partial_{t} \boldsymbol{\eta}_{h}^{n+1}\right) \cdot \boldsymbol{v}_{h}-\gamma \frac{\mu}{h} \int_{\Sigma}\left(\boldsymbol{u}_{h}^{n}-\partial_{t} \boldsymbol{\eta}_{h}^{n+1}\right) \cdot \boldsymbol{w}_{h} \\
& +S\left(p_{h}^{n+1}, q_{h}\right)=\int_{\Gamma_{\text {out }}} \boldsymbol{g}\left(t_{n+1}\right) \cdot \boldsymbol{v}_{h},
\end{aligned}
$$

for all $\left(\boldsymbol{v}_{h}, q_{h}, \boldsymbol{w}_{h}, \dot{\boldsymbol{w}}_{h}\right) \in V_{h} \times Q_{h} \times X_{h} \times X_{h}$. Equivalently, in a more compact form,

$$
A_{\mathrm{S}}\left[\left(\boldsymbol{U}_{h}^{n+1}, \boldsymbol{\Theta}_{h}^{n+1}\right),\left(\boldsymbol{V}_{h}, \boldsymbol{W}_{h}\right)\right]=\int_{\Gamma \text { out }} \boldsymbol{g}^{n+1} \cdot \boldsymbol{v}_{h},
$$

for all $\left(\boldsymbol{v}_{h}, q_{h}, \boldsymbol{w}_{h}, \dot{\boldsymbol{w}}_{h}\right) \in V_{h} \times Q_{h} \times X_{h} \times X_{h}$, where the stabilized explicit coupling bilinear form $A_{\mathrm{S}}$ is given by

$$
A_{\mathrm{S}}\left[\left(\boldsymbol{U}_{h}^{n+1}, \boldsymbol{\Theta}_{h}^{n+1}\right),\left(\boldsymbol{V}_{h}, \boldsymbol{W}_{h}\right)\right] \stackrel{\text { def }}{=} A_{\mathrm{E}}\left[\left(\boldsymbol{U}_{h}^{n+1}, \boldsymbol{\Theta}_{h}^{n+1}\right),\left(\boldsymbol{V}_{h}, \boldsymbol{W}_{h}\right)\right]+S\left(p_{h}^{n+1}, q_{h}\right) \text {. }
$$

On the other hand, from 22$]$, we obtain

$$
\begin{aligned}
A_{\mathrm{S}}\left[\left(\boldsymbol{U}_{h}^{n+1}, \boldsymbol{\Theta}_{h}^{n+1}\right),\left(\boldsymbol{V}_{h}, \boldsymbol{W}_{h}\right)\right]= & A_{\mathrm{I}}\left[\left(\boldsymbol{U}_{h}^{n+1}, \boldsymbol{\Theta}_{h}^{n+1}\right),\left(\boldsymbol{V}_{h}, \boldsymbol{W}_{h}\right)\right] \\
& +\gamma \frac{\mu}{h} \int_{\Sigma}\left(\boldsymbol{u}_{h}^{n+1}-\boldsymbol{u}_{h}^{m}\right) \cdot \boldsymbol{w}_{h} \\
& +\int_{\Sigma}\left(\boldsymbol{\sigma}\left(\boldsymbol{u}_{h}^{n+1}, p_{h}^{n+1}\right) \boldsymbol{n}-\boldsymbol{\sigma}\left(\boldsymbol{u}_{h}^{n}, p_{h}^{n}\right) \boldsymbol{n}\right) \cdot\left(\boldsymbol{v}_{h}-\boldsymbol{w}_{h}\right) \\
& +\frac{\gamma_{0} h}{\gamma \mu} \int_{\Sigma}\left(p_{h}^{n+1}-p_{h}^{n}\right) q_{h} .
\end{aligned}
$$

\subsection{Stability analysis}

In this section we will prove that the dissipative character of the Nitsche's penalty coupling term in combination with (30) makes the explicit scheme (14)(15) stable, for $\gamma_{0}$ sufficiently large and under the CFL-like condition (24). 
Theorem 5.2 Assume that the fluid-structure system is isolated, i.e. $\overline{\boldsymbol{u}}=\mathbf{0}$ and $\boldsymbol{g}=\mathbf{0}$. Let $\left\{\left(\boldsymbol{u}_{h}^{n+1}, p_{h}^{n+1}, \boldsymbol{\eta}_{h}^{n+1}, \dot{\boldsymbol{\eta}}_{h}^{n+1}\right)\right\}_{0 \leq n \leq N-1}$ denote the solution of (31)(32). Under the following conditions

$$
\begin{aligned}
& \gamma \geq 256 C_{\mathrm{TI}}, \\
& \gamma \delta t \leq C_{\Sigma} h, \\
& \gamma_{0} \geq 8,
\end{aligned}
$$

the following energy estimate holds, for $1 \leq n \leq N$,

$$
\begin{aligned}
& E^{n}+3 \delta t \frac{\gamma \mu}{h}\left\|\boldsymbol{u}_{h}^{n}\right\|_{0, \Sigma}^{2}+3 \frac{\gamma_{0} h}{\gamma \mu} \delta t\left\|p_{h}^{n}\right\|_{0, \Sigma}^{2} \\
& \quad \leq 6 E^{0}+3 C_{\Sigma} \mu\left\|\boldsymbol{u}_{h}^{0}\right\|_{0, \Sigma}^{2}+\frac{3 \mu}{4}\left\|\boldsymbol{\epsilon}\left(\boldsymbol{u}_{h}^{0}\right)\right\|_{0, \Omega_{\mathrm{f}}}^{2}+3 \frac{\gamma_{0} h}{\gamma \mu} \delta t\left\|p_{h}^{0}\right\|_{0, \Sigma}^{2} \cdot
\end{aligned}
$$

In other words, the stabilized explicit coupling scheme (31)-32 is conditionally stable in the energy norm.

Proof. We proceed by first testing 33 with

$$
\left(\boldsymbol{v}_{h}, q_{h}, \boldsymbol{w}_{h}, \dot{\boldsymbol{w}}_{h}\right)=\left(\boldsymbol{u}_{h}^{n+1}, p_{h}^{n+1}, \partial_{\delta t} \boldsymbol{\eta}_{h}^{n+1}, \partial_{\delta t} \dot{\boldsymbol{\eta}}_{h}^{n+1}\right) .
$$

Then, using (34), multiplying by $\delta t$, replacing index $n$ by $m$ and summing over $0 \leq m \leq n-1$ and using the stability analysis of the (unstabilized) explicit coupling scheme (Lemma 4.2), under conditions 35$)_{1,2}$ we have

$$
\begin{gathered}
E^{n}+3 \delta t \frac{\gamma \mu}{h}\left\|\boldsymbol{u}_{h}^{n}\right\|_{0, \Sigma}^{2}+\underbrace{6 \gamma_{0} \frac{h}{\gamma \mu} \delta t \sum_{m=0}^{n-1} \int_{\Sigma}\left(p_{h}^{m+1}-p_{h}^{m}\right) p_{h}^{m+1}}_{T_{1}} \\
\leq 6 E^{0}+3 C_{\Sigma} \mu\left\|\boldsymbol{u}_{h}^{0}\right\|_{0, \Sigma}^{2}+\frac{3 \mu}{4}\left\|\boldsymbol{\epsilon}\left(\boldsymbol{u}_{h}^{0}\right)\right\|_{0, \Omega_{\mathrm{f}}}^{2}+\underbrace{24 \frac{h}{\gamma \mu} \delta t \sum_{m=0}^{n-1}\left\|p_{h}^{m+1}-p_{h}^{m}\right\|_{0, \Sigma}^{2}}_{T_{2}} .
\end{gathered}
$$

In order to prove the stability of the scheme, we simply show that the term $T_{1}$ can absorb the term $T_{2}$. Indeed, note that for the first term we have

$$
T_{1}=3 \gamma_{0} \frac{h}{\gamma \mu} \delta t\left(\left\|p_{h}^{n}\right\|_{0, \Sigma}^{2}-\left\|p_{h}^{0}\right\|_{0, \Sigma}^{2}\right)+3 \gamma_{0} \frac{h}{\gamma \mu} \delta t \sum_{m=0}^{n-1}\left\|p_{h}^{m+1}-p_{h}^{m}\right\|_{0, \Sigma}^{2} .
$$

Therefore, by inserting this expression in (37), term $T_{2}$ in 37 can be cancelled by the dissipative term in $T_{1}$ under condition $[35]_{3}$, which completes the proof.

We conclude this section with a series of remarks.

Remark 5.3 According to Theorem 5.2, the stability of the stabilized explicit coupling scheme is independent of the added-mass effect: the fluid-solid density ratio $\rho_{\mathrm{f}} / \rho_{\mathrm{s}}$ and the length of the domain. This is a major advantage compared 
to the standard explicit-coupling, whose (in)stability precisely relies on these quantities, irrespectively of the discretization parameters (see [7, [16]). Finally, let us note that, contrarily to $[35]_{3}$, the stability condition announced in [4], for the original stabilized explicit coupling scheme (see Remark 5.1), made the time penalty parameter depend on the geometry of the domain (at least theoretically).

Remark 5.4 The proof of Theorem 5.2 is based, exclusively, on the dissipation due to the Nitsche coupling and the time pressure penalization term. As a result, our stability result is independent of the dissipative features of the fluid and solid time discretization schemes. This is a significant progress with respect to the stability result stated in [12], for a semi-implicit coupling scheme, whose proof purely depends on the dissipative properties of the solid time discretization scheme (a leap-frog scheme). On the other hand, as regards the fluid timediscretization, one can use, for instance, a neutrally stable second order scheme.

Remark 5.5 Because of their common structure, we have used the terminology CFL for the stability condition $(35)_{2}$. However, we must stress that their nature and impact on the numerical solution are completely different. Actually, from the proof of Lemma 4.2. one can notice that $\sqrt{35})_{2}$ does not arise from bounding amplification factors (see also the Remark 5.6), which is indeed the case for the classical CFL condition.

Remark 5.6 Failing to satisfy condition $(35)_{2}$, indicates that $C_{\Sigma}$ is unbounded, that is to say,

$$
\frac{\gamma \delta t}{h} \rightarrow \infty
$$

On the other hand, from the proofs of Lemma 4.2 and Theorem 5.2, we have that

$$
\frac{\gamma \delta t}{h} \mu\left\|\boldsymbol{u}_{h}^{n}\right\|_{0, \Sigma}^{2} \leq 2 E^{0}+\frac{\gamma \delta t}{h} \mu\left\|\boldsymbol{u}_{h}^{0}\right\|_{0, \Sigma}^{2}+\frac{\mu}{4}\left\|\boldsymbol{\epsilon}\left(\boldsymbol{u}_{h}^{0}\right)\right\|_{0, \Omega_{\mathrm{f}}}^{2}+\frac{\gamma_{0} h}{\gamma \mu} \delta t\left\|p_{h}^{0}\right\|_{0, \Sigma}^{2},
$$

for all $0 \leq n \leq N-1$. As a result, the combination of (38) and (39) yields

$$
\left\|\boldsymbol{u}_{h}^{n}\right\|_{0, \Sigma} \rightarrow\left\|\boldsymbol{u}_{h}^{0}\right\|_{0, \Sigma}, \quad 0 \leq n \leq N-1
$$

In summary, taking a very large value of $C_{\Sigma}$ forces the interface fluid velocity to satisfy (40) and, therefore, introduces an artificial unphysical decoupling between the fluid and the solid.

\subsection{Stabilization and artificial compressibility}

In this paragraph we motivate the role of the time-penalty term 30 as a quasiincompressible approximation at the discrete level. First, note that the stabilization term can be rewritten as

$$
S\left(p_{h}^{n+1}, q_{h}\right) \stackrel{\text { def }}{=} \frac{\gamma_{0} h \delta t}{\gamma \mu} \int_{\Sigma} \partial_{\delta t} p_{h}^{n+1} q_{h}
$$

As a result, the discrete continuity equation in 32 is given by

$$
\frac{\gamma_{0} h \delta t}{\gamma \mu} \int_{\Sigma} \partial_{\delta t} p_{h}^{n+1} q_{h}+\int_{\Omega_{\mathrm{f}}} q_{h} \boldsymbol{\nabla} \cdot \boldsymbol{u}_{h}^{n+1}=\int_{\Sigma}\left(\boldsymbol{u}_{h}^{n+1}-\partial_{\delta t} \boldsymbol{\eta}_{h}^{n+1}\right) \cdot \boldsymbol{n} q_{h},
$$

$\mathrm{RR} \mathrm{n}^{\circ} 6445$ 
for all $q_{h} \in Q_{h}$, which corresponds to the discrete counterpart of the modified continuity equation

$$
c_{\delta t, \Sigma} \partial_{t} p+\nabla \cdot \boldsymbol{u}=0, \quad \text { in } \Omega_{\mathrm{f}} .
$$

Here, $c_{\delta t, \Sigma} \stackrel{\text { def }}{=} \gamma_{0} \delta t h(\gamma \mu)^{\frac{1}{2}} \boldsymbol{\delta}_{\Sigma}$ can be thought as an artificial compressibility parameter, where $\boldsymbol{\delta}_{\Sigma}$ stands for the Dirac's measure on the surface $\Sigma$.

We can, therefore, interpret the stabilization of the explicit coupling scheme (30) as a quasi-incompressible approximation. Let us note that, the artificial compressibility $c_{\delta t, \Sigma}$ is purely restricted to the wall $\Sigma$, through the Dirac's measure $\boldsymbol{\delta}_{\Sigma}$. In practice, it is limited to the first layer of fluid mesh elements from the interface $\Sigma$. Formally, $c_{\delta t, \Sigma}$ goes to zero as long as $\delta t \rightarrow 0$ or $h \rightarrow 0$. Actually, we shall see in the next paragraph that the penalty term 30 is of order $O\left(h^{\frac{1}{2}} \delta t^{\frac{1}{2}}\right)$, and hence consistent with the original implicit scheme.

The role of the divergence-free constraint in the fluid-structure coupling has already been outlined in the literature. Let us cite, for instance, the works 36 , 40, 35, 22, where a pseudo-compressibility is introduced in order to accelerate the convergence of fixed-point iterations towards the solution of an implicit coupling scheme. Here, the compressibility term vanishes at convergence and the artificial compressibility parameter is chosen so as to optimize efficiency (it only depends on physical quantities) and not for consistency or stability purposes.

\subsection{Weak consistency and error estimates}

A full convergence analysis of the proposed method is beyond the scope of the present paper, although it can be performed under suitable assumptions on the stabilization parameters and on the regularity of the exact solution. We will however show how the truncation error of the artificially introduced penalty term influences the precision. Let $\boldsymbol{\theta}_{h}^{n} \stackrel{\text { def }}{=} \boldsymbol{u}_{h}^{n}-\pi_{h} \boldsymbol{u}\left(t_{n}\right), y_{h}^{n} \stackrel{\text { def }}{=} p_{h}^{n}-\pi_{h} p\left(t_{n}\right)$, $\boldsymbol{\xi}_{h}^{n} \stackrel{\text { def }}{=} \boldsymbol{\eta}_{h}^{n}-\pi_{h} \boldsymbol{\eta}\left(t_{n}\right)$ and $\dot{\boldsymbol{\xi}}_{h}^{n} \stackrel{\text { def }}{=} \dot{\boldsymbol{\eta}}_{h}^{n}-\pi_{h} \dot{\boldsymbol{\eta}}\left(t_{n}\right)$ be the discrete errors where $\pi_{h}$ denotes some suitable interpolation operator.

We introduce the following discrete error functional

$$
\begin{aligned}
& \mathcal{E}^{n}=\left(\frac{\rho_{\mathrm{f}}}{2}\left\|\boldsymbol{\theta}_{h}^{n}\right\|_{0, \Omega_{\mathrm{f}}}^{2}+\frac{\rho_{\mathrm{s}}}{2}\left\|\dot{\boldsymbol{\xi}}_{h}^{n}\right\|_{0, \Omega_{\mathrm{s}}}^{2}+\frac{1}{2} a^{s}\left(\boldsymbol{\xi}_{h}^{n}, \boldsymbol{\xi}_{h}^{n}\right)+\frac{3 \mu}{4} \sum_{m=0}^{n-1} \delta t\left\|\boldsymbol{\epsilon}\left(\boldsymbol{\theta}_{h}^{m+1}\right)\right\|_{0, \Omega_{\mathrm{f}}}^{2}\right. \\
& \left.+3 \delta t \frac{\gamma \mu}{h}\left\|\boldsymbol{\theta}_{h}^{n}\right\|_{0, \Sigma}^{2}+3 \frac{\gamma_{0} h \delta t}{\gamma \mu}\left\|y_{h}^{n}\right\|_{0, \Sigma}^{2}+\frac{3 \gamma \mu}{4 h} \delta t \sum_{m=0}^{n-1}\left\|\boldsymbol{\theta}_{h}^{m+1}-\partial_{\delta t} \boldsymbol{\xi}_{h}^{m+1}\right\|_{0, \Sigma}^{2}\right)^{\frac{1}{2}} .
\end{aligned}
$$

A formal error estimate for our formulation can be obtained using the stability result of Theorem 5.2, on the discrete error quantities $\boldsymbol{\xi}_{h}^{n}, \boldsymbol{\theta}_{h}^{n}$, and $y_{h}^{n}$, followed by an application of the (modified) Galerkin orthogonality, which gives rise to the following upper bound of the error:

$$
\left(\mathcal{E}^{n}\right)^{2} \leq C\left[\left(\mathcal{E}^{0}\right)^{2}+\tau_{1}+\tau_{2}\right] .
$$

The terms on the right hand side consists of $\mathcal{E}^{0}$, that are terms measuring the error in initial data, $\tau_{1}$ denotes the terms appearing from the time and space discretization and the splitting error and, finally, $\tau_{2}$ is the non-consistency introduced by the time penalty operator on the pressure. In this work we will 
only discuss $\tau_{2}$. One may then show that the exact form of the stabilization contribution to the error representation is

$$
\delta t \sum_{m=0}^{n-1} S\left(\pi_{h} p\left(t_{m+1}\right), y_{h}^{m+1}\right)=\frac{\gamma_{0} h}{\gamma \mu} \delta t \sum_{m=0}^{n-1} \int_{\Sigma}\left(\pi_{h} p\left(t_{m+1}\right)-\pi_{h} p\left(t_{m}\right)\right) y_{h}^{m+1} .
$$

Using a Taylor expansion, the truncation error is given by

$$
\left|\pi_{h} p\left(t_{m+1}\right)-\pi_{h} p\left(t_{m}\right)\right| \leq \int_{t^{m}}^{t^{m+1}}\left|\partial_{t} \pi_{h} p\right| \leq \delta t^{\frac{1}{2}}\left(\int_{t^{m}}^{t^{m+1}}\left|\partial_{t} \pi_{h} p\right|^{2}\right)^{\frac{1}{2}}
$$

Finally, by applying the estimate 43 to 42 , it follows that

$$
\delta t \sum_{m=0}^{n-1} S\left(\pi_{h} p\left(t_{m+1}\right), y_{h}^{m+1}\right) \leq \underbrace{\frac{\gamma_{0} T}{2 \gamma \mu} h \delta t\left\|\partial_{t} \pi_{h} p\right\|_{0, \Sigma \times(0, T)}^{2}}_{\tau_{2}}+\frac{\gamma_{0} h}{2 \gamma \mu} \frac{\delta t^{2}}{T} \sum_{m=0}^{n-1}\left\|y_{h}^{m+1}\right\|_{0, \Sigma}^{2} .
$$

Estimate 41 follows by applying a Gronwall's lemma on the second term, noting that

$$
\frac{\gamma_{0} h}{2 \gamma \mu} \frac{\delta t^{2}}{T} \sum_{m=0}^{n-1}\left\|y_{h}^{m+1}\right\|_{0, \Sigma}^{2} \leq \frac{\delta t}{T} \sum_{m=0}^{n-1} \mathcal{E}^{m+1} .
$$

Using now the local trace-inverse inequality (5), followed by the $H^{1}$-stability of the interpolation operator we may conclude

$$
\tau_{2} \leq \frac{C_{\mathrm{TI}}}{2} \frac{\gamma_{0} T}{\gamma \mu} h \delta t\left\|\partial_{t} \nabla p\right\|_{0, \Omega_{\mathrm{f}} \times(0, T)}^{2}
$$

Some observations are then in order:

- The consistency order of the stabilization term is $O\left(h^{\frac{1}{2}} \delta t^{\frac{1}{2}}\right)$, provided $\partial_{t} p \in H^{1}\left(\Omega_{\mathrm{f}} \times(0, T)\right)$. Note that the impact of the stabilization term on the accuracy of the scheme decreases when we refine in time or in space.

- From the stability condition $(35)_{2}$, we have $\delta t=O(h)$. Therefore, the consistency of the stabilization term rates as $O(h)$, which is optimal if piece-wise linear finite elements are used for the discretization of the fluid velocities or the structure displacements.

- Since we expect the error in time to be dominated by the contribution from the stabilization term, the error bound (41) should then take the form

$$
\mathcal{E}^{n} \leq C\left[\mathcal{E}^{0}+\left(\frac{\gamma_{0} T}{\gamma \mu}\right)^{\frac{1}{2}} h^{\frac{1}{2}} \delta t^{\frac{1}{2}}\right] .
$$

In particular, since $\delta t=O(h)$, we should have

$$
\mathcal{E}^{n} \leq C\left[\mathcal{E}^{0}+\frac{\left(C_{\Sigma} \gamma_{0} T\right)^{\frac{1}{2}}}{\mu^{\frac{1}{2}} \gamma} h\right]
$$


- The stabilization gives rise to a factor $T^{\frac{1}{2}}$ in the error estimates (44) and 45. Therefore, in the most unfavorable case, the error grows linearly with $T^{\frac{1}{2}}$.

- If only the time discretization is considered, i.e. $h$ is fixed, the error estimate (44) anticipates a sub-optimal $O\left(\delta t^{\frac{1}{2}}\right)$ convergence rate. The next paragraph addresses this issue.

\subsection{Improving time accuracy: defect-correction iterations}

In this paragraph we propose to recover optimal accuracy in time, namely $O(\delta t)$, by performing a few iterations based on the defect-correction method. The iterative defect-correction method (see e.g. 37]) is an iterative procedure which aims at increasing the accuracy of a numerical solution, without mesh refinement. More precisely, to approximate the solution $x^{*}$ of the equation

$$
F(x)=0,
$$

in terms of a simplified (typically less accurate) problem

$$
\tilde{F}(x)=0,
$$

the defect-correction iteration consists in solving, for $k \geq 0$ :

$$
\tilde{F}\left(x_{k+1}\right)=\tilde{F}\left(x_{k}\right)-F\left(x_{k}\right) .
$$

We now propose to make use of this iterations in order to improve the accuracy of the stabilized explicit scheme (Algorithm 5). More precisely, we want to recover the accuracy of the (underlying) implicit scheme (18) with a few correction iterations (per time step) on the stabilized explicit scheme.

In terms of the above notations we then set:

$$
\begin{aligned}
& x \stackrel{\text { def }}{=}\left(\boldsymbol{U}_{h}^{n+1}, \boldsymbol{\Theta}_{h}^{n+1}\right), \\
&\left\langle F(x),\left(\boldsymbol{V}_{h}, \boldsymbol{W}_{h}\right)\right\rangle \stackrel{\text { def }}{=} A_{\mathrm{I}}\left[\left(\boldsymbol{U}_{h}^{n+1}, \boldsymbol{\Theta}_{h}^{n+1}\right),\left(\boldsymbol{V}_{h}, \boldsymbol{W}_{h}\right)\right]-\int_{\Gamma_{\text {out }}} \boldsymbol{g}\left(t_{n+1}\right) \cdot \boldsymbol{v}_{h}, \\
&\left\langle\tilde{F}(x),\left(\boldsymbol{V}_{h}, \boldsymbol{W}_{h}\right)\right\rangle \stackrel{\text { def }}{=} A_{\mathrm{S}}\left[\left(\boldsymbol{U}_{h}^{n+1}, \boldsymbol{\Theta}_{h}^{n+1}\right),\left(\boldsymbol{V}_{h}, \boldsymbol{W}_{h}\right)\right]-\int_{\Gamma_{\text {out }}} \boldsymbol{g}\left(t_{n+1}\right) \cdot \boldsymbol{v}_{h} .
\end{aligned}
$$

Thus, the defect difference $\left\langle\tilde{F}\left(x_{k}\right)-F\left(x_{k}\right),\left(\boldsymbol{V}_{h}, \boldsymbol{W}_{h}\right)\right\rangle$ in 46 is given by

$$
\begin{aligned}
\frac{\gamma_{0} h}{\gamma \mu} \int_{\Sigma}\left(p_{h}^{n+1, k}-p_{h}^{n}\right) q_{h}+\gamma \frac{\mu}{h} \int_{\Sigma}\left(\boldsymbol{u}_{h}^{n+1, k}-\boldsymbol{u}_{h}^{n}\right) \cdot \dot{\boldsymbol{w}}_{h} \\
+\int_{\Sigma}\left(\boldsymbol{\sigma}\left(\boldsymbol{u}_{h}^{n+1, k}, p_{h}^{n+1, k}\right) \boldsymbol{n}-\boldsymbol{\sigma}\left(\boldsymbol{u}_{h}^{n}, p_{h}^{n}\right) \boldsymbol{n}\right) \cdot\left(\boldsymbol{v}_{h}-\boldsymbol{w}_{h}\right) .
\end{aligned}
$$

As a result, the stabilized explicit scheme (Algorithm 5) combined with $K \geq$ 0 defect-correction iterations, (46), leads to the following iterative procedure: Here, we used the notation $\partial_{\delta t} X^{n+1, q} \stackrel{\text { def }}{=}\left(X^{n+1, q}-X^{n}\right) / \delta t$.

Remark 5.7 Note that, for $K=0$ (i.e. with no corrections) the above algorithm reduces exactly to the original stabilized explicit coupling scheme (Algorithm 5). 
Stabilized explicit coupling with $K \geq 0$ corrections.

- Time loop: For $0 \leq n \leq N-1$ let $\left(\boldsymbol{u}_{h}^{n+1,0}, p_{h}^{n+1,0}\right) \stackrel{\text { def }}{=}\left(\boldsymbol{u}_{h}^{n}, p_{h}^{n}\right)$.

1. Correction loop: For $0 \leq k \leq K$, solve:

(a) Solid sub-step: Find $\left(\boldsymbol{\eta}_{h}^{n+1, k+1}, \dot{\boldsymbol{\eta}}_{h}^{n+1, k+1}\right) \in X_{h} \times X_{h}$ such that

$$
\begin{array}{r}
A_{\mathrm{s}, \delta t}\left(\left(\boldsymbol{\eta}_{h}^{n+1, k+1}, \dot{\boldsymbol{\eta}}_{h}^{n+1, k+1}\right),\left(\boldsymbol{w}_{h}, \dot{\boldsymbol{w}}_{h}\right)\right)+\gamma \frac{\mu}{h} \int_{\Sigma} \partial_{\delta t} \boldsymbol{\eta}_{h}^{n+1, k+1} \cdot \boldsymbol{w}_{h} \\
\quad=\gamma \frac{\mu}{h} \int_{\Sigma} \boldsymbol{u}_{h}^{n+1, k} \cdot \boldsymbol{w}_{h}-\int_{\Sigma} \boldsymbol{\sigma}\left(\boldsymbol{u}_{h}^{n+1, k}, p_{h}^{n+1, k}\right) \boldsymbol{n} \cdot \boldsymbol{w}_{h}, \quad(47)
\end{array}
$$

for all $\left(\dot{\boldsymbol{w}}_{h}, \boldsymbol{w}_{h}\right) \in X_{h} \times X_{h}$.

(b) Fluid sub-step: Find $\left(\boldsymbol{u}_{h}^{n+1, k+1}, p_{h}^{n+1, k+1}\right) \in W_{h} \times Q_{h}$ with $\boldsymbol{u}_{h}^{n+1, k+1}=\overline{\boldsymbol{u}}_{h}\left(t_{n+1}\right)$ on $\Gamma^{\text {in }}$, such that

$$
\begin{gathered}
A_{\mathrm{f}, \delta t}\left(\left(\boldsymbol{u}_{h}^{n+1, k+1}, p_{h}^{n+1, k+1}\right),\left(\boldsymbol{v}_{h}, q_{h}\right)\right)-\int_{\Sigma} \boldsymbol{u}_{h}^{n+1, k+1} \cdot \boldsymbol{\sigma}\left(\alpha \boldsymbol{v}_{h},-q_{h}\right) \boldsymbol{n} \\
+\gamma \frac{\mu}{h} \int_{\Sigma} \boldsymbol{u}_{h}^{n+1, k+1} \cdot \boldsymbol{v}_{h}+\frac{\gamma_{0} h}{\gamma \mu} \int_{\Sigma} p_{h}^{n+1, k+1} q_{h}=\int_{\Sigma} \boldsymbol{\sigma}\left(\boldsymbol{u}_{h}^{n+1, k}, p_{h}^{n+1, k}\right) \boldsymbol{n} \cdot \boldsymbol{v}_{h} \\
-\int_{\Sigma} \partial_{\delta t} \boldsymbol{\eta}_{h}^{n+1, k+1} \cdot \boldsymbol{\sigma}\left(\alpha \boldsymbol{v}_{h},-q_{h}\right) \boldsymbol{n}+\gamma \frac{\mu}{h} \int_{\Sigma} \partial_{\delta t} \boldsymbol{\eta}_{h}^{n+1, k+1} \cdot \boldsymbol{v}_{h} \\
+\frac{\gamma_{0} h}{\gamma \mu} \int_{\Sigma} p_{h}^{n+1, k} q_{h}+\int_{\Gamma_{\text {out }}} \boldsymbol{g}\left(t_{n+1}\right) \cdot \boldsymbol{v}_{h},
\end{gathered}
$$

for all $\left(\boldsymbol{v}_{h}, q_{h}\right) \in V_{h} \times Q_{h}$.

\section{Update solution:}

$$
\boldsymbol{u}_{h}^{n+1}=\boldsymbol{u}_{h}^{n+1, K+1}, \quad p_{h}^{n+1}=p_{h}^{n+1, K+1}, \quad \boldsymbol{\eta}_{h}^{n+1}=\boldsymbol{\eta}_{h}^{n+1, K+1} .
$$


One of the main features of the above algorithm is that, after $K \geq 0$ corrections, the solution is expected to be of order $O\left(\delta t+\delta t^{\frac{K+1}{2}}\right)$. So, one defectcorrection iteration is enough to recover first order optimality. This will be illustrated with numerical results in the next section. The corresponding theoretical convergence analysis of $47-48$ is beyond the scope of this paper. We refer the interested reader to [23, where error estimates of defect-corrections methods are obtained in a different framework.

Remark 5.8 The optimal number of correction iterations depends on the accuracy of the underlying implicit coupling scheme. For instance, if a CrankNicolson time discretization is used for the fluid and a mid-point rule for the structure, from $O\left(\delta t^{2}+\delta t^{\frac{K+1}{2}}\right)$, second order accuracy is expected after three correction iterations $(K=3)$.

\subsection{The non-linear case}

In this paragraph we provide an extension of the explicit coupling scheme with $K \geq 0$ corrections, Algorithm 5.4, to the case of a general non-linear fluidstructure problem, involving a viscous incompressible fluid. We assume that the fluid is described by the incompressible Navier-Stokes equations, using the ALE (arbitrary Lagrangian-Eulerian) formalism (see e.g. [10]), and the structure by the non-linear elastodynamics equations.

Let $\Omega=\Omega_{\mathrm{f}} \cup \Omega_{\mathrm{s}}$ be a reference configuration of the system. The current configuration of the fluid domain, $\Omega^{\mathrm{f}}(t)$, is parametrized by the ALE map $\mathcal{A} \stackrel{\text { def }}{=} \boldsymbol{I}_{\Omega_{\mathrm{f}}}+\boldsymbol{\eta}_{\mathrm{f}}$ as $\Omega_{\mathrm{f}}(t)=\mathcal{A}\left(\Omega_{\mathrm{f}}, t\right)$, where $\boldsymbol{\eta}_{\mathrm{f}}: \Omega_{\mathrm{f}} \times \mathbb{R}^{+} \rightarrow \mathbb{R}^{d}$ stands for the displacement of the fluid domain. In practice, $\boldsymbol{\eta}_{\mathrm{f}}=\operatorname{Ext}\left(\left.\boldsymbol{\eta}\right|_{\Sigma}\right)$, where $\operatorname{Ext}(\cdot)$ denotes any reasonable lifting operator from the (reference) interface $\Sigma$ into the (reference) fluid domain $\Omega_{\mathrm{f}}$. For instance, an harmonic lifting operator is used in the numerical experiments reported in paragraph 6.2

The non-linear fluid-structure problem under consideration reads as follows: Find the fluid velocity $\boldsymbol{u}: \Omega_{\mathrm{f}} \times \mathbb{R}^{+} \rightarrow \mathbb{R}^{d}$, the pressure $p: \Omega_{\mathrm{f}} \times \mathbb{R}^{+} \rightarrow \mathbb{R}^{d}$ and the solid displacement $\boldsymbol{\eta}: \Omega_{\mathrm{s}} \times \mathbb{R}^{+} \rightarrow \mathbb{R}^{d}$ such that

$$
\begin{aligned}
& \left\{\begin{array}{rlrl}
\left.\rho_{\mathrm{f}} \frac{\partial \boldsymbol{u}}{\partial t}\right|_{\mathcal{A}}+\rho_{\mathrm{f}}(\boldsymbol{u}-\boldsymbol{w}) \cdot \nabla \boldsymbol{u}-\nabla \cdot \boldsymbol{\sigma}(\boldsymbol{u}, p) & =\mathbf{0}, & \text { in } \quad \Omega_{\mathrm{f}}(t), \\
\boldsymbol{\nabla} \cdot \boldsymbol{u} & =0, & \text { in } \quad \Omega_{\mathrm{f}}(t), \\
\boldsymbol{u} & =\overline{\boldsymbol{u}}, & & \text { on } \quad \Gamma^{\text {in }}, \\
\boldsymbol{\sigma}(\boldsymbol{u}, p) \boldsymbol{n} & =\boldsymbol{g}, & & \text { on } \quad \Gamma^{\text {out }},
\end{array}\right. \\
& \left\{\begin{array}{rlrl}
\rho_{\mathrm{s}} \frac{\partial^{2} \boldsymbol{\eta}}{\partial t^{2}}-\boldsymbol{\nabla} \cdot \boldsymbol{\Pi}(\boldsymbol{\eta}) & =0, & \text { in } \quad \Omega_{\mathrm{s}} \\
\boldsymbol{\eta}=\mathbf{0}, & \text { on } \quad \Gamma^{\mathrm{d}} \\
\boldsymbol{\Pi}(\boldsymbol{\eta}) \boldsymbol{n}_{\mathrm{s}}=\mathbf{0}, & \text { on } \quad \Gamma^{\mathrm{n}}
\end{array}\right.
\end{aligned}
$$

with the interface coupling conditions

$$
\left\{\begin{array}{l}
\boldsymbol{\eta}_{\mathrm{f}}=\operatorname{Ext}\left(\left.\boldsymbol{\eta}\right|_{\Sigma}\right), \quad \boldsymbol{w}=\frac{\partial \boldsymbol{\eta}_{\mathrm{f}}}{\partial t}, \quad \text { in } \quad \Omega_{\mathrm{f}} \quad \Omega_{\mathrm{f}}(t)=\left(\boldsymbol{I}_{\Omega_{\mathrm{f}}}+\boldsymbol{\eta}_{\mathrm{f}}\right)\left(\Omega_{\mathrm{f}}\right), \\
\boldsymbol{u}=\frac{\partial \boldsymbol{\eta}}{\partial t}, \quad \text { on } \quad \Sigma(t), \\
\boldsymbol{\Pi}(\boldsymbol{\eta}) \boldsymbol{n}_{\mathrm{s}}=-J_{\mathrm{f}} \boldsymbol{\sigma}(\boldsymbol{u}, p) \boldsymbol{F}_{\mathrm{f}}^{-T} \boldsymbol{n}, \quad \text { on } \quad \Sigma .
\end{array}\right.
$$


where $\left.\frac{\partial}{\partial t}\right|_{\mathcal{A}}$ represents the ALE time derivative, $\boldsymbol{\Pi}(\boldsymbol{\eta})$ the first Piola-Kirchhoff stress tensor of the structure (related to the displacement $\boldsymbol{\eta}$ through an appropriate constitutive law), $\boldsymbol{F}_{\mathrm{f}} \stackrel{\text { def }}{=} \boldsymbol{\nabla} \mathcal{A}$ the fluid domain gradient of deformation and $J_{\mathrm{f}} \stackrel{\text { def }}{=} \operatorname{det} \boldsymbol{F}_{\mathrm{f}}$ the Jacobian. Note that a field defined in the reference fluid domain, $\Omega_{\mathrm{f}}$, is evaluated in the current fluid domain, $\Omega_{\mathrm{f}}(t)$, by composition with $\mathcal{A}^{-1}(\cdot, t)$.

We propose to approximate the non-linear coupled problem (49)-(51) using the framework of the stabilized explicit coupling scheme (Algorithms 5 and 5.4). With this aim, we first redefine $A_{\mathrm{f}, \delta t}$ and $A_{\mathrm{s}, \delta t}$ according to the new fluid and solid variational formulations. The fluid equations are discretized in time using a semi-implicit backward Euler's scheme, with an explicit treatment of the fluid domain motion, whereas for for the structure we use a Newmark's scheme. As a result, we have

$$
\begin{aligned}
A_{\mathrm{f}, \delta t}\left(\left(\boldsymbol{u}_{h}^{n+1}, p_{h}^{n+1}\right),\left(\boldsymbol{v}_{h}, q_{h}\right)\right) \stackrel{\text { def }}{=} & \rho_{\mathrm{f}} \\
\delta t & \left(\int_{\Omega_{\mathrm{f}}^{n}} \boldsymbol{u}_{h}^{n+1} \cdot \boldsymbol{v}_{h}-\int_{\Omega_{\mathrm{f}}^{n-1}} \boldsymbol{u}_{h}^{n} \cdot \boldsymbol{v}_{h}\right) \\
& +\frac{\rho_{\mathrm{f}}}{2} \int_{\Omega_{\mathrm{f}}^{n}}\left(\boldsymbol{\nabla} \cdot \boldsymbol{u}_{h}^{n}\right) \boldsymbol{u}_{h}^{n+1} \cdot \boldsymbol{v}_{h} \\
& -\rho_{\mathrm{f}} \int_{\Omega_{\mathrm{f}}^{n}}\left(\boldsymbol{\nabla} \cdot \boldsymbol{w}_{h}^{n}\right) \boldsymbol{u}_{h}^{n+1} \cdot \boldsymbol{v}_{h} \\
& +\rho_{\mathrm{f}} \int_{\Omega_{\mathrm{f}}^{n}}\left(\boldsymbol{u}_{h}^{n}-\boldsymbol{w}_{h}^{n}\right) \cdot \nabla \boldsymbol{u}_{h}^{n+1} \cdot \boldsymbol{v}_{h} \\
& +\int_{\Omega_{\mathrm{f}}^{n}} \boldsymbol{\sigma}\left(\boldsymbol{u}_{h}^{n+1}, p_{h}^{n+1}\right): \boldsymbol{\epsilon}\left(\boldsymbol{v}_{h}\right) \\
& +\int_{\Omega_{\mathrm{f}}^{n}} q_{h} \boldsymbol{\nabla} \cdot \boldsymbol{u}_{h}^{n+1}, \\
A_{\mathrm{s}, \delta t}\left(\left(\boldsymbol{\eta}_{h}^{n+1}, \dot{\boldsymbol{\eta}}_{h}^{n+1}\right),\left(\boldsymbol{w}_{h}, \dot{\boldsymbol{w}}_{h}\right)\right) \stackrel{\text { def }}{=} & \rho_{\mathrm{s}} \int_{\Omega_{\mathrm{s}}} \partial_{\delta t} \dot{\boldsymbol{\eta}}_{h}^{n+1} \cdot \boldsymbol{w}_{h} \\
& +\frac{1}{2} \int_{\Omega_{\mathrm{s}}}\left(\boldsymbol{\Pi}\left(\boldsymbol{\eta}_{h}^{n+1}\right)+\boldsymbol{\Pi}\left(\boldsymbol{\eta}_{h}^{n}\right)\right): \nabla \boldsymbol{w}_{h} \\
& +\rho_{\mathrm{s}} \int_{\Omega_{\mathrm{s}}}\left(\frac{\dot{\boldsymbol{\eta}}_{h}^{n+1}+\dot{\boldsymbol{\eta}}_{h}^{m}}{2}-\partial_{\delta t} \boldsymbol{\eta}_{h}^{n+1}\right) \cdot \dot{\boldsymbol{w}}_{h} .
\end{aligned}
$$

The second and third terms in (52) come, respectively, from the conservative treatment of the ALE time derivative and the linearization of the convective term. Note that, due to the explicit treatment of the fluid domain motion, all the variational terms in $A_{\mathrm{f}, \delta t}$ are defined over a known domain, either $\Omega_{\mathrm{f}}^{n}$ or $\Omega_{\mathrm{f}}^{n-1}$.

The stabilized explicit scheme, with $K \geq 0$ defect-correction iterations, applied to the non-linear coupled problem $(49)-(51)$, is then given by the following iterative procedure. Due to the explicit treatment of the the fluid domain geometry, the Nitsche's interface terms and the explicit coupling stabilization are evaluated over a know position of the interface, $\Sigma^{n}$. On the other hand, the Nitsche's penalty term in the solid-subproblem (53) is also integrated over $\Sigma^{n}$, instead of over the reference interface configuration $\Sigma$, which is someway unusual in Lagrangian structural mechanics. 
Stabilized explicit coupling with $K \geq 0$ corrections (non-linear version).

- Time loop: For $0 \leq n \leq N-1$ let $\left(\boldsymbol{u}_{h}^{n+1,0}, p_{h}^{n+1,0}\right) \stackrel{\text { def }}{=}\left(\boldsymbol{u}_{h}^{n}, p_{h}^{n}\right)$.

1. Correction loop: For $0 \leq k \leq K$, solve:

(a) Solid sub-step: Find $\left(\boldsymbol{\eta}_{h}^{n+1, k+1}, \dot{\boldsymbol{\eta}}_{h}^{n+1, k+1}\right) \in X_{h} \times X_{h}$ such that

$$
\begin{gathered}
A_{\mathrm{s}, \delta t}\left(\left(\boldsymbol{\eta}_{h}^{n+1, k+1}, \dot{\boldsymbol{\eta}}_{h}^{n+1, k+1}\right),\left(\boldsymbol{w}_{h}, \dot{\boldsymbol{w}}_{h}\right)\right)+\gamma \frac{\mu}{h} \int_{\Sigma^{n}} \partial_{\delta t} \boldsymbol{\eta}_{h}^{n+1, k+1} \cdot \boldsymbol{w}_{h} \\
=\gamma \frac{\mu}{h} \int_{\Sigma^{n}} \boldsymbol{u}_{h}^{n+1, k} \cdot \boldsymbol{w}_{h}-\int_{\Sigma^{n}} \boldsymbol{\sigma}\left(\boldsymbol{u}_{h}^{n+1, k}, p_{h}^{n+1, k}\right) \boldsymbol{n} \cdot \boldsymbol{w}_{h}
\end{gathered}
$$

for all $\left(\dot{\boldsymbol{w}}_{h}, \boldsymbol{w}_{h}\right) \in X_{h} \times X_{h}$.

(b) Fluid sub-step: Find $\left(\boldsymbol{u}_{h}^{n+1, k+1}, p_{h}^{n+1, k+1}\right) \in W_{h} \times Q_{h}$ with $\boldsymbol{u}_{h}^{n+1, k+1}=\overline{\boldsymbol{u}}_{h}\left(t_{n+1}\right)$ on $\Gamma^{\text {in }}$, such that

$$
\begin{gathered}
A_{\mathrm{f}, \delta t}\left(\left(\boldsymbol{u}_{h}^{n+1, k+1}, p_{h}^{n+1, k+1}\right),\left(\boldsymbol{v}_{h}, q_{h}\right)\right)-\int_{\Sigma^{n}} \boldsymbol{u}_{h}^{n+1, k+1} \cdot \boldsymbol{\sigma}\left(\alpha \boldsymbol{v}_{h},-q_{h}\right) \boldsymbol{n} \\
+\gamma \frac{\mu}{h} \int_{\Sigma^{n}} \boldsymbol{u}_{h}^{n+1, k+1} \cdot \boldsymbol{v}_{h}+\frac{\gamma_{0} h}{\gamma \mu} \int_{\Sigma^{n}} p_{h}^{n+1, k+1} q_{h}=\int_{\Sigma^{n}} \boldsymbol{\sigma}\left(\boldsymbol{u}_{h}^{n+1, k}, p_{h}^{n+1, k}\right) \boldsymbol{n} \cdot \boldsymbol{v}_{h} \\
-\int_{\Sigma^{n}} \partial_{\delta t} \boldsymbol{\eta}_{h}^{n+1, k+1} \cdot \boldsymbol{\sigma}\left(\alpha \boldsymbol{v}_{h},-q_{h}\right) \boldsymbol{n}+\gamma \frac{\mu}{h} \int_{\Sigma^{n}} \partial_{\delta t} \boldsymbol{\eta}_{h}^{n+1, k+1} \cdot \boldsymbol{v}_{h} \\
+\frac{\gamma_{0} h}{\gamma \mu} \int_{\Sigma^{n}} p_{h}^{n+1, k} q_{h}+\int_{\Gamma_{\text {out }}} \boldsymbol{g}\left(t_{n+1}\right) \cdot \boldsymbol{v}_{h},
\end{gathered}
$$

for all $\left(\boldsymbol{v}_{h}, q_{h}\right) \in V_{h} \times Q_{h}$.

\section{Update solution:}

$$
\boldsymbol{u}^{n+1}=\boldsymbol{u}^{n+1, K+1}, \quad p^{n+1}=p^{n+1, K+1}, \quad \boldsymbol{\eta}^{n+1}=\boldsymbol{\eta}^{n+1, K+1} .
$$

3. Update fluid domain (mesh):

$$
\begin{aligned}
& \boldsymbol{\eta}_{\mathrm{f}}^{n+1}=\operatorname{Ext}\left(\left.\boldsymbol{\eta}^{n+1}\right|_{\Sigma}\right), \quad \boldsymbol{w}^{n+1}=\partial_{\delta t} \boldsymbol{\eta}_{\mathrm{f}}^{n+1} \text { in } \Omega_{\mathrm{f}}, \\
& \Omega_{\mathrm{f}}^{n+1}=\left(\boldsymbol{I}_{\Omega_{\mathrm{f}}}+\boldsymbol{\eta}_{\mathrm{f}}^{n+1}\right)\left(\Omega_{\mathrm{f}}\right) .
\end{aligned}
$$




\section{Numerical experiments}

In this section we illustrate the properties of the stabilized explicit coupling scheme, with $K \geq 0$ corrections, by performing a series of numerical experiments.

\subsection{A linear two-dimensional test case}

We consider a simplified version of the numerical experiment reported in [30, 15, by coupling the 2D Stokes equations with the linear elasticity equations, i.e. the simplified coupled problem (1)-(3). The fluid domain is given by $\Omega_{\mathrm{f}} \stackrel{\text { def }}{=}$

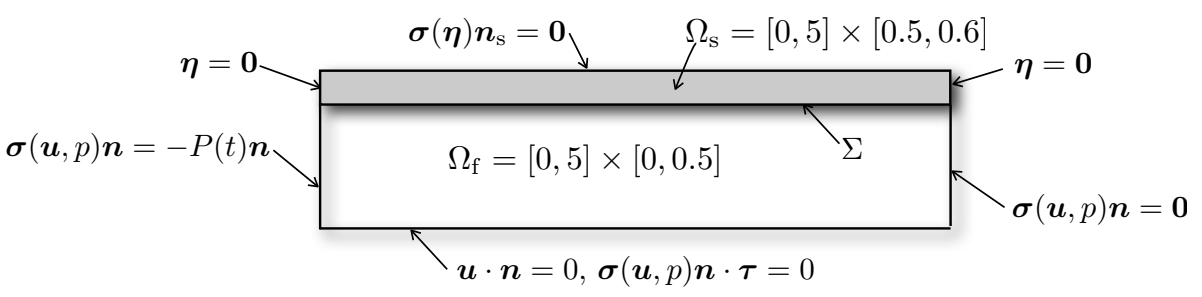

Figure 2: Geometrical description and boundary conditions

$[0,5] \times[0,0.5]$ and the solid domain by $\Omega_{\mathrm{s}} \stackrel{\text { def }}{=}[0,5] \times[0.5,0.6]$, all the space units are in $\mathrm{cm}$.

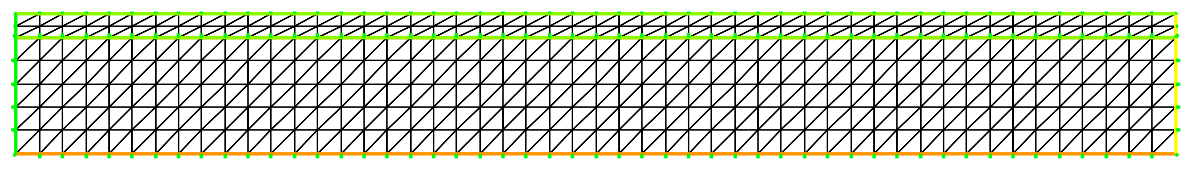

Figure 3: Fluid and solid finite element meshes

Both systems, the fluid and the structure, are initially at rest. We impose, between $x=0$ and $x=5$, an over pressure of $P=10^{4} \mathrm{dyne} / \mathrm{cm}^{2}$ during $5 \times 10^{-3} \mathrm{~s}$. The structure is clamped on $x=0$ and $x=5$ and zero traction is applied on $y=0.6$ (see Figure 2). The fluid physical parameters are given by $\rho_{\mathrm{f}}=1.1 \mathrm{~g} / \mathrm{cm}^{d}$ and $\mu=0.035$ poise. For the solid we have $\rho_{\mathrm{s}}=1.2 \mathrm{~g} / \mathrm{cm}^{3}$, $E=3 \times 10^{8}$ dyne $/ \mathrm{cm}^{2}$ for the Young modulus and a Poisson ratio of $\nu=0.3$.

The fluid space discretization is based on the Taylor-Hood $\left(\mathbb{P}_{2} / \mathbb{P}_{1}\right)$ finite element, a standard $\mathbb{P}_{1}$-continuous discretization is used for the structure. The mesh size was set to $h=0.1$ (see Figure 3 ) and the time step to $\delta t=10^{-4} \mathrm{~s}$. We consider the symmetric Nitsche's formulation, i.e. $\alpha=1$. All the 2D numerical computations have been performed with FreeFem $++[21$.

Regarding the free parameters $\gamma$ and $\gamma_{0}$, we have taken $\gamma=2500$, which is within the range predicted by the stability condition $(35)_{1}$. For the time penalty parameter $\gamma_{0}$, the numerical tests showed that the the stability condition $353_{3}$ over estimates its critical value. In practice, we can take a lower value without compromising stability, $\gamma_{0}=1$, which is more convenient in terms of accuracy. For the motivation of this choice, we refer to the discussion below on the sensitivity results reported in Tables 1 and 2 . The same values are used for the non-linear 3D numerical tests reported in paragraph $\$ 6.2$. 


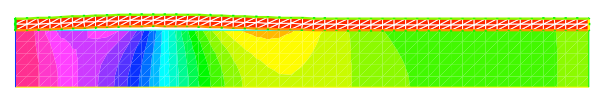

(a) $t=0.0025 \mathrm{~s}$

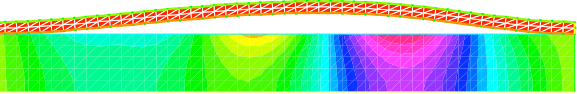

(c) $t=0.01 \mathrm{~s}$

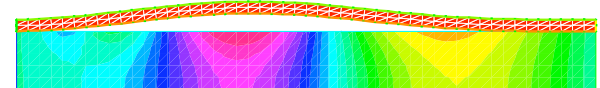

(b) $t=0.075 \mathrm{~s}$

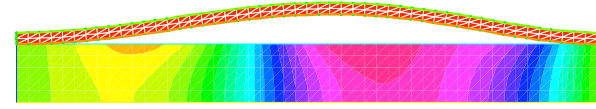

(d) $t=0.015 \mathrm{~s}$

Figure 4: Stabilized explicit coupling $\left(K=0, \alpha=1, \gamma=2500, \gamma_{0}=1\right)$ : snapshots of the pressure and solid deformation (exaggerated) at four time instants.
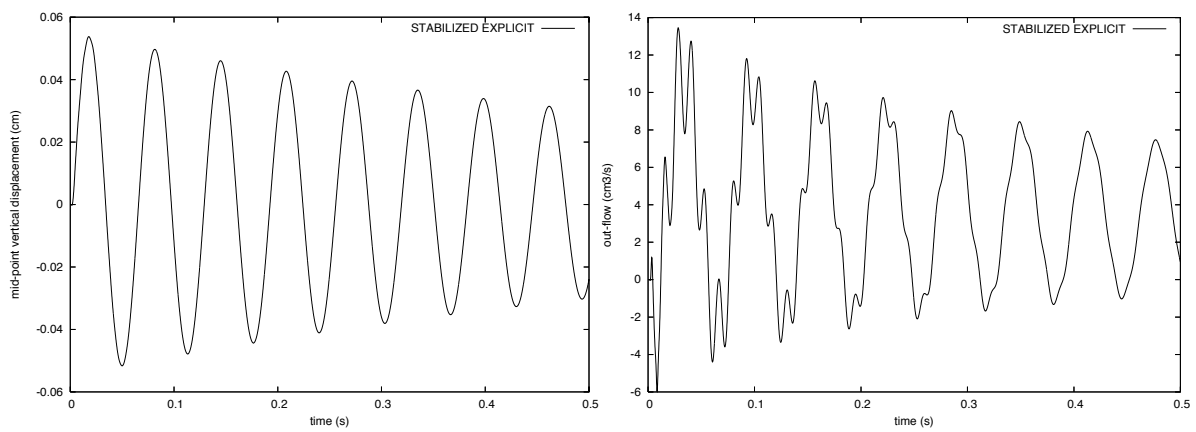

Figure 5: Stabilized explicit coupling $\left(K=0, \alpha=1, \gamma=2500, \gamma_{0}=1\right)$ : interface mid-point $y$-displacement (left) and out-flow rate (right)

Let us consider, first, the stabilized explicit coupling scheme without correction (Algorithm 5 or, equivalently, Algorithm 5.4 with $K=0$ ). In Figure 4 we have reported the computed pressure contours and the solid deformed configuration at four time instants. The interface mid-point $y$-displacement and the out-flow are depicted in Figure 5. As predicted by Theorem 5.2 the numerical solution is stable, and we can observe a pressure wave that propagates through the fluid domain (see e.g [30, 9]).

We now investigate the impact of the added-mass effect on the stability of the stabilized explicit coupling scheme. To this aim, we first increase the fluidsolid density ratio $\rho_{\mathrm{f}} / \rho_{\mathrm{s}}$. We keep constant the solid density, $\rho_{\mathrm{s}}=1.2$, and take $\rho_{\mathrm{f}}$ ranging from $10^{-2}$ to $10^{3}$. The corresponding interface mid-point vertical displacements are shown in Figure 6(left). We make also vary the length of the domain, $L$, from 5 to $40 \mathrm{~cm}$. The corresponding out-flow rates are reported in Figure 6(right). As predicted by Theorem 5.2 (see also Remark 5.3), the numerical solution remains stable in both cases, irrespectively of the amount of added-mass effect.

Let us turn our attention to the accuracy of the numerical solution provided by the stabilized explicit coupling scheme without correction $(K=0)$. In Figure 7. we compare the interface mid-point $y$-displacement and the out-flow obtained with the stabilized explicit coupling (without correction) and a standard implicit coupling scheme (in which the kinematic condition $(3)_{1}$ is strongly enforced). The latter being solved using Aitken's relaxed Dirichlet-Neumann iterations (see 

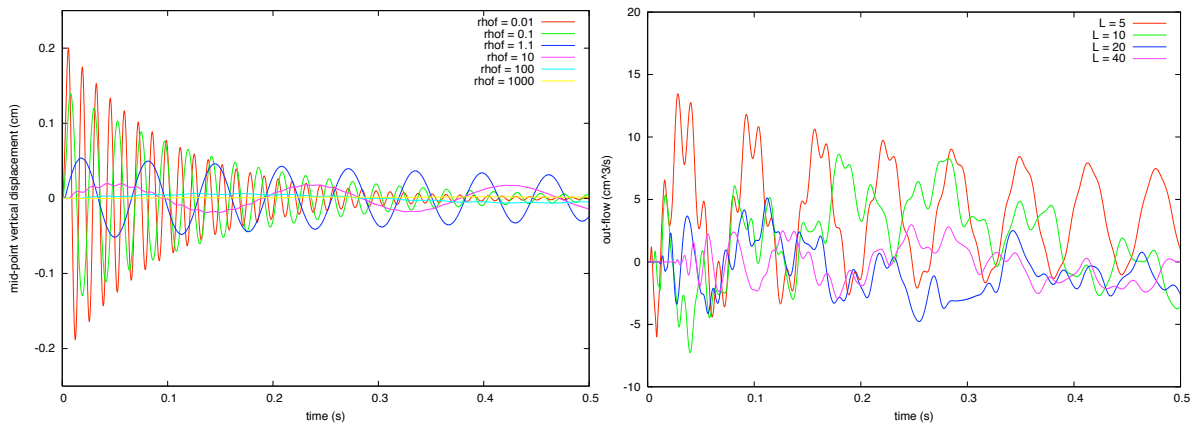

Figure 6: Stabilized explicit coupling $\left(K=0, \alpha=1, \gamma=2500, \gamma_{0}=1\right)$ : Interface mid-point $y$-displacement for different values of the fluid-solid density ratio $\rho_{\mathrm{f}} / \rho_{\mathrm{s}}$ (left), out-flow rate for different values of the vessel length $L$ (right)
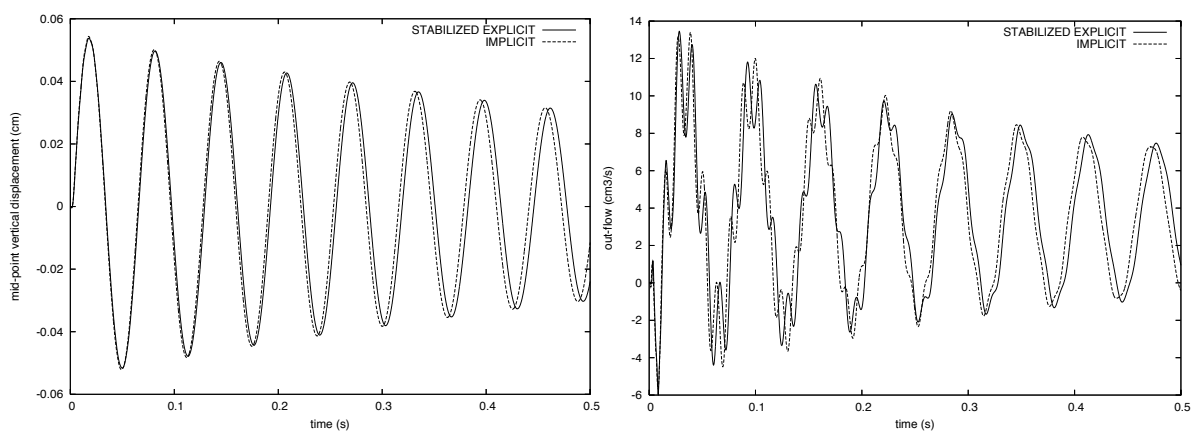

Figure 7: Interface mid-point $y$-displacement (left) and out-flow rate (right): comparison of stabilized explicit coupling $\left(K=0, \alpha=1, \gamma=2500, \gamma_{0}=1\right)$ and implicit coupling (strongly enforced kinematic conditions)
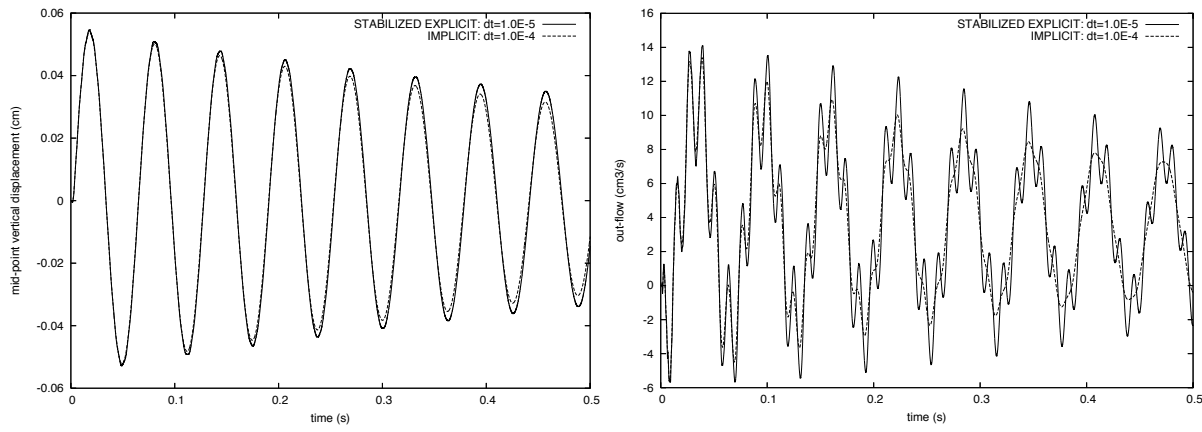

Figure 8: Interface mid-point $y$-displacement (left) and out-flow rate (right), obtained with the stabilized explicit coupling $\left(K=0, \alpha=1, \gamma=2500, \gamma_{0}=1\right)$ for $\delta t=10^{-5}$, and with the implicit coupling (strongly enforced kinematic condition) for $\delta t=10^{-4}$

e.g. [27]), with an average of 22 iterations, per time step, for a residual tolerance of $10^{-7}$ on the interface displacement. 
Figure 7 shows that the numerical solution provided by the stabilized explicit coupling scheme (without correction) is able to capture the global behavior of the solution given by implicit coupling scheme. Let us stress that two different coupling formulations are used for the explicit and the implicit coupling. Some local features of the implicit coupling solution, in the out-flow rate, are however not fully resolved by the explicit coupling. This reduced accuracy is in agreement with the formal consistency analysis performed in Paragraph $\$ 5.3$. Indeed, for the stabilized explicit coupling scheme the convergence rate (in time) is expected to be $O\left(\delta t^{\frac{1}{2}}\right)$, whereas for the implicit scheme an optimal $O(\delta t)$ is assumed. Figure 8 shows that the stabilized explicit coupling scheme, for $\delta t=10^{-5}$, reaches the accuracy of the implicit coupling scheme, with $\delta t=10^{-4}$. Clearly, improving the accuracy of the stabilized explicit solution by reducing the time step is inefficient in practice.

\begin{tabular}{|r||l|l|l|l|l|l|l|}
\hline$\gamma_{0}$ & 1 & 100 & 500 & 1000 & 2500 & 6250 & 12500 \\
\hline \hline 0.25 & $\mathrm{X}$ & $\mathrm{X}$ & $\mathrm{X}$ & $\mathrm{X}$ & $\mathrm{X}$ & $\mathrm{X}$ & $\mathrm{X}$ \\
\hline 0.5 & $\mathrm{X}$ & $\mathrm{X}$ & $\mathrm{X}$ & $\mathrm{X}$ & $\mathrm{X}$ & $\mathrm{X}$ & $\mathrm{X}$ \\
\hline 1 & $\mathrm{X}$ & 0.8992 & 0.4733 & 0.3687 & 0.3180 & 0.3981 & 0.4925 \\
\hline 2 & $\mathrm{X}$ & 1.0468 & 0.6382 & 0.4807 & 0.3785 & 0.4025 & 0.4941 \\
\hline 4 & $\mathrm{X}$ & 1.2918 & 0.8046 & 0.6449 & 0.4550 & 0.4490 & 0.4941 \\
\hline 8 & $\mathrm{X}$ & 1.5330 & 0.9603 & 0.8066 & 0.5374 & 0.4729 & 0.5021 \\
\hline 16 & $\mathrm{X}$ & 1.8987 & 1.2284 & 0.9649 & 0.7052 & 0.5440 & 0.5485 \\
\hline
\end{tabular}

Table 1: Stabilized explicit coupling $(K=0)$ with the symmetric formulation $(\alpha=1)$ : out-flow relative errors

\begin{tabular}{|r||l|l|l|l|l|l|l|}
\hline$\gamma_{0}$ & 1 & 100 & 500 & 1000 & 2500 & 6250 & 12500 \\
\hline \hline 0.25 & $\mathrm{X}$ & $\mathrm{X}$ & $\mathrm{X}$ & $\mathrm{X}$ & $\mathrm{X}$ & $\mathrm{X}$ & $\mathrm{X}$ \\
\hline 0.5 & $\mathrm{X}$ & $\mathrm{X}$ & $\mathrm{X}$ & $\mathrm{X}$ & $\mathrm{X}$ & $\mathrm{X}$ & $\mathrm{X}$ \\
\hline 1 & $\mathrm{X}$ & 0.9112 & 0.4737 & 0.3684 & 0.3178 & 0.3981 & 0.4925 \\
\hline 2 & $\mathrm{X}$ & 1.0552 & 0.6403 & 0.4810 & 0.3783 & 0.4025 & 0.4941 \\
\hline 4 & $\mathrm{X}$ & 1.3237 & 0.8070 & 0.6460 & 0.4549 & 0.4490 & 0.4941 \\
\hline 8 & $\mathrm{X}$ & 1.5517 & 0.9664 & 0.8078 & 0.5377 & 0.4729 & 0.5020 \\
\hline 16 & $\mathrm{X}$ & 1.9139 & 1.2327 & 0.9664 & 0.7057 & 0.5441 & 0.5484 \\
\hline
\end{tabular}

Table 2: Stabilized explicit coupling $(K=0)$ with the non-symmetric formulation $(\alpha=-1)$ : out-flow relative errors

We shall see below that, according to Paragraph \$5.4 one defect-correction iteration $(K=1)$ is enough to recover the accuracy of the implicit coupling scheme. In the meantime, we propose to illustrate, through a series of numerical computations, the impact of $\gamma$ and $\gamma_{0}$ on the accuracy of the numerical solution, and their role in the stability condition (35) and the error estimate (44). 
To this aim, we compute the relative out-flow $l^{\infty}$-errors:

$$
\frac{\max _{0 \leq n \leq N-1}\left|Q_{\mathrm{STAB}-\mathrm{EXPLICIT}}^{n+1}-Q_{\mathrm{IMPLICIT}}^{n+1}\right|}{\max _{0 \leq n \leq N-1}\left|Q_{\text {IMPLICIT }}^{n+1}\right|},
$$

of the stabilized explicit coupling scheme $(K=0)$ with respect to the implicit coupling scheme (strongly enforced kinematic condition). The corresponding error, for different choices of $\gamma, \gamma_{0}$, with $\delta t=10^{-4}$ and $h=0.1$, are given in Table 1, for the symmetric formulation $(\alpha=1)$, and in Table 2 for the nonsymmetric $(\alpha=-1)$. The symbol "X" indicates that numerical instability can be obtained for a specific choice of $\delta t$ and $h$.

The results reported in Tables 1 and 2 show that the numerical solution, provided by the explicit coupling scheme, is stable as long as we take a sufficiently large value of $\gamma$ and $\gamma_{0}$. In other words, we recover the stability conditions (35) 1,3. For both formulations, symmetric and non-symmetric, the numerical solution becomes unstable for sufficiently small values of $\gamma$ or $\gamma_{0}$, which is also in agreement with the stability conditions $35 p_{1,3}$. Note that this conditions over-estimate the critical stability values of $\gamma$ and $\gamma_{0}$.

In terms of accuracy, Tables 1 and 2 show that the symmetric and nonsymmetric formulations give similar results, particularly for large values of $\gamma$. As predicted by (44), for a given $\gamma$, the highest accuracy corresponds to the lowest value of $\gamma_{0}$. Moreover, for a given $\gamma_{0}$, increasing $\gamma$ (up to a certain level) improves the accuracy of the solution. In particular, we can observe that the error grows almost linearly with $\gamma_{0}^{\frac{1}{2}}$ and $\gamma^{-\frac{1}{2}}$. Note that, for a sufficiently large $\gamma$, the stability condition $(35)_{2}$ leads to a very large value of $C_{\Sigma}$ (since $h, \delta t$ are fixed). Therefore, according to Remark 5.6, this may deteriorate the accuracy of the numerical solution, as it can be noticed in Tables 1 and 2. This shows, in particular, that the procedure of improving the accuracy by increasing the Nitsche penalty $\gamma$ is limited by the impact of $C_{\Sigma}$ on the energy estimate 36 . and, as result, on the error bound 45.
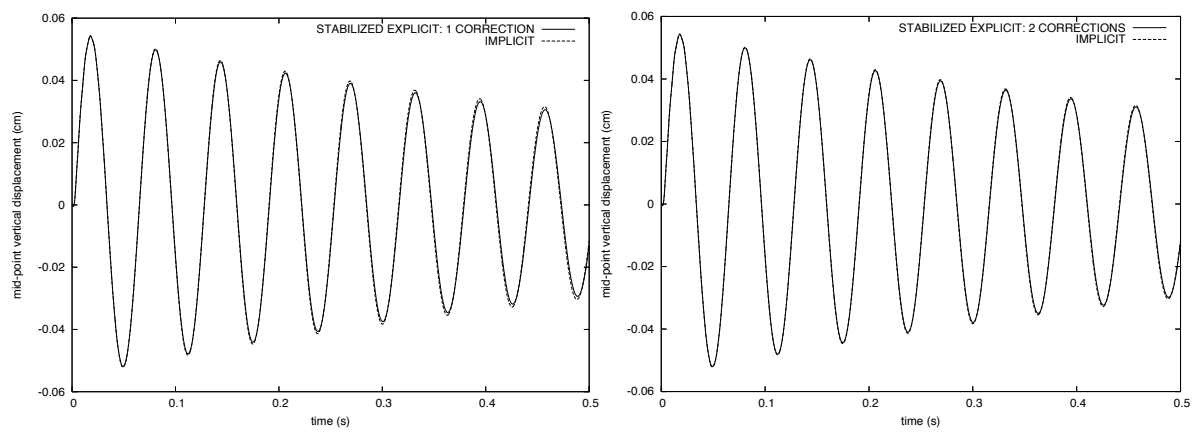

Figure 9: Interface mid-point $y$-displacement: stabilized explicit coupling with correction $\left(K=1,2, \alpha=1, \gamma=2500, \gamma_{0}=1\right)$ and implicit coupling (strongly enforced kinematic condition)

Finally, in order to improve the time accuracy of the solution, we consider the stabilized explicit coupling scheme with one or two corrections $(K=1,2)$. In Figures 9 and 10 we report a comparison with the implicit coupling scheme. 

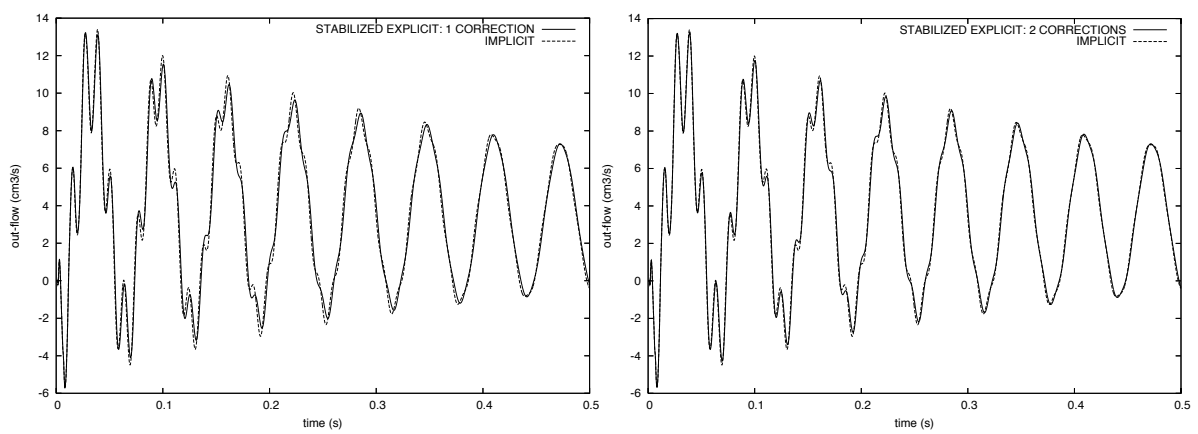

Figure 10: Out-flow: stabilized explicit coupling with correction $(K=1,2$, $\alpha=1, \gamma=2500, \gamma_{0}=1$ ) and implicit coupling (strongly enforced kinematic condition)
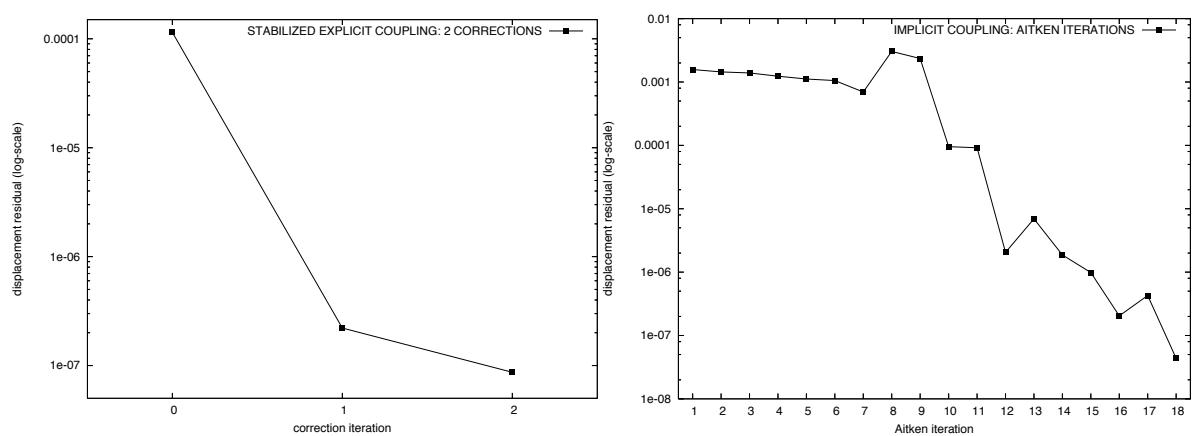

Figure 11: $L^{2}$-norm of the displacement residual (log-scale) at time $t=0.5 \mathrm{~s}$ : stabilized explicit coupling $\left(\alpha=1, \gamma=2500, \gamma_{0}=1\right)$ with $K=2$ corrections (left), implicit coupling (strongly enforced kinematic condition) solved via Aitken's relaxed iterations (right)

After one correction step, the stabilized explicit coupling scheme achieves first order accuracy $O(\delta t)$ : the enhanced accuracy is clearly visible (see Figure 7), in particular, in the outflow rate. After two correction steps it provides a solution almost undistinguishable from the implicit scheme solution. This is a clear indication that, once the same order is reached (i.e after one correction), further corrections are superfluous. This feature is also illustrated in Figure 11 (left), where we report the displacement residual reduction for each correction iteration, at the last time step. Note that achieving optimality, i.e. the first correction, involves the largest residual reduction. For comparison purposes, Figure 11(right) shows the corresponding residual history provided by the iterative procedure used for solving the implicit coupling (Aitken's relaxed iterations).

\subsection{A non-linear three-dimensional test case}

We investigate the stability, accuracy and efficiency of Algorithm 5.5, the nonlinear variant of the stabilized explicit coupling scheme with correction. 


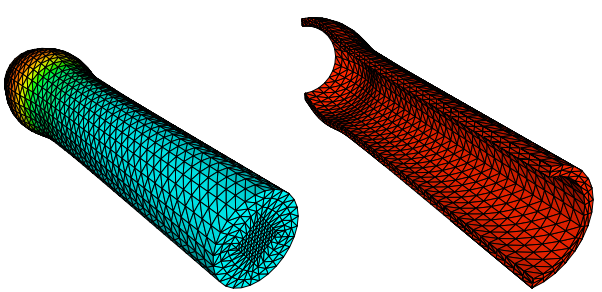

(a) $t=0.0025 \mathrm{~s}$

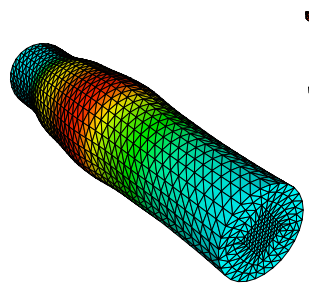

(c) $t=0.0075 \mathrm{~s}$

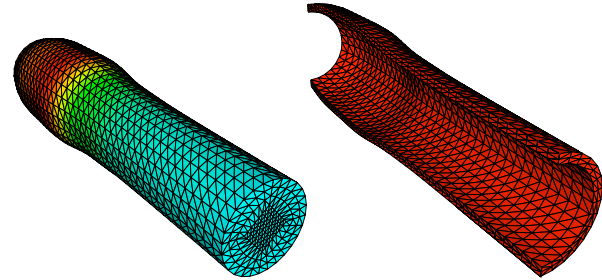

(b) $t=0.005 \mathrm{~s}$

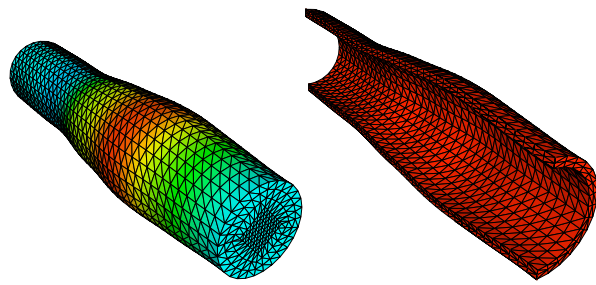

(d) $t=0.01 \mathrm{~s}$

Figure 12: Stabilized explicit coupling without correction $(K=0, \alpha=1, \gamma=$ $2500, \gamma_{0}=1$ ): snapshots of the pressure and solid deformation (exaggerated) at four time instants

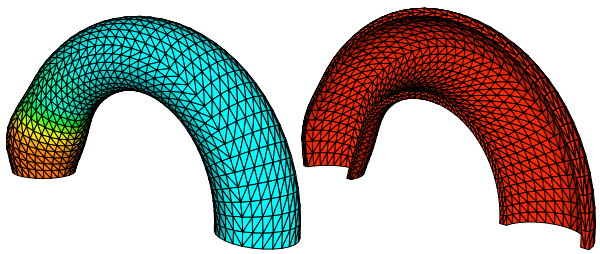

(a) $t=0.0025 \mathrm{~s}$

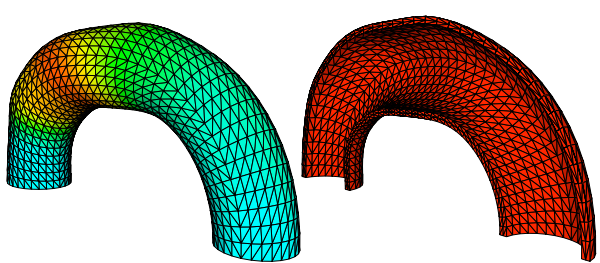

(c) $t=0.0075 \mathrm{~s}$

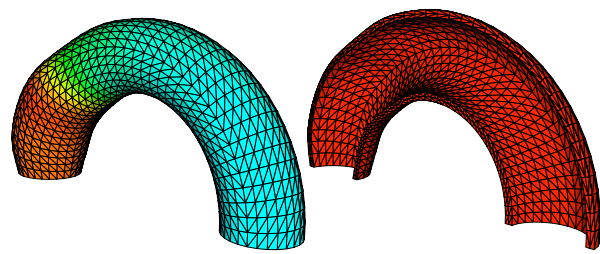

(b) $t=0.005 \mathrm{~s}$

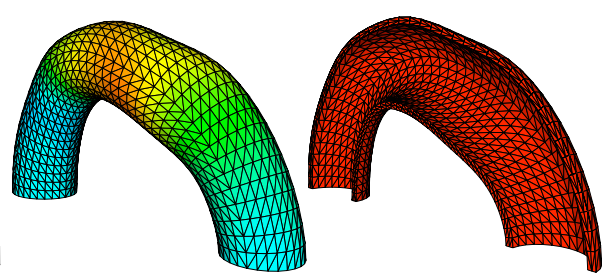

(d) $t=0.01 \mathrm{~s}$

Figure 13: Stabilized explicit coupling without correction $(K=0, \alpha=1, \gamma=$ $2500, \gamma_{0}=1$ ): snapshots of the pressure and solid deformation (exaggerated) at four time instants

We consider the 3D non-linear version of the numerical experiment analyzed in the previous paragraph (see e.g. [17, 12]). Accordingly with (49)-(51), the structure is described by the non-linear elastodynamics equations (St. VenantKirchhoff material) and the fluid by the incompressible Navier-Stokes equations with an ALE formulation. We consider, as in [14, two different geometries: 
1. a straight vessel of radius $0.5 \mathrm{~cm}$ and length $5 \mathrm{~cm}$,

2. a curved vessel of radius $0.5 \mathrm{~cm}$ with curvature ratio $0.25 \mathrm{~cm}^{-1}$.

The surrounding structure has a thickness of $0.1 \mathrm{~cm}$. The physical parameters are the following:

- Fluid: viscosity $\mu=0.035$ poise, density $\rho_{\mathrm{f}}=1 \mathrm{~g} / \mathrm{cm}^{3}$,

- Solid: density $\rho_{\mathrm{s}}=1.2 \mathrm{~g} / \mathrm{cm}^{3}$, Young modulus $E=3 \times 10^{6}$ dynes $/ \mathrm{cm}^{2}$ and Poisson ratio $\nu=0.3$.
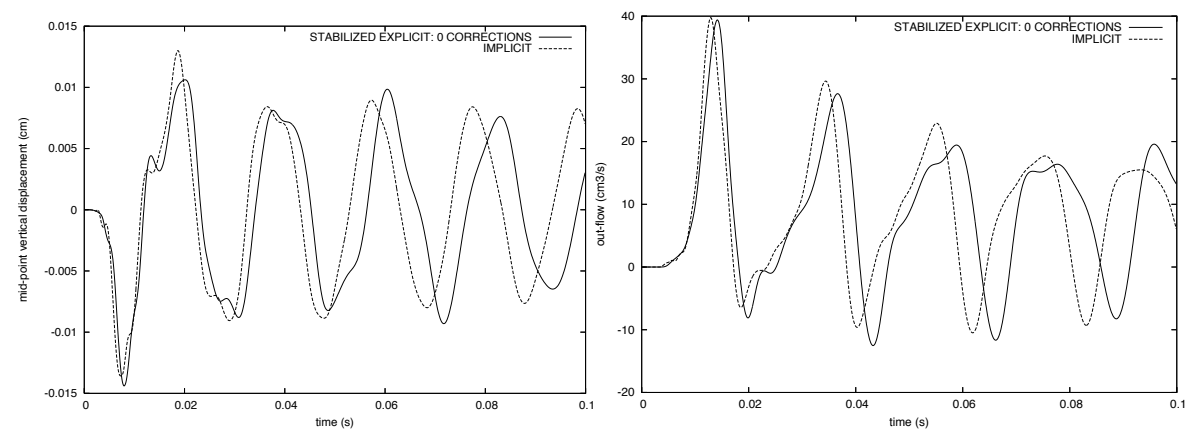

Figure 14: Straight vessel. Stabilized explicit coupling without correction $(K=$ $\left.0, \alpha=1, \gamma=2500, \gamma_{0}=1\right)$ : interface mid-point $y$-displacement (left), and out-flow rate (right)
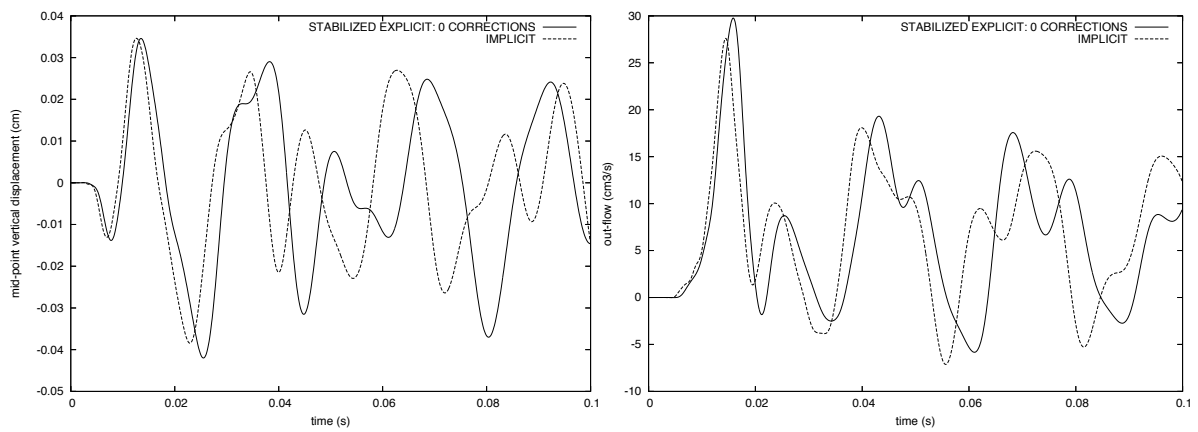

Figure 15: Curved vessel. Stabilized explicit coupling without correction $(K=$ $\left.0, \alpha=1, \gamma=2500, \gamma_{0}=1\right)$ : interface mid-point $y$-displacement (left), and out-flow rate (right)

Both systems, the fluid and the structure, are initially at rest. The structure is clamped at the inlet and the outlet. An over pressure of $1.3332 \times$ $10^{4}$ dynes $/ \mathrm{cm}^{2}$ is imposed, on the inlet boundary, during $5 \times 10^{-3}$ seconds. The fluid equations are discretized using $\mathbb{P}_{1} / \mathbb{P}_{1}$-continuous stabilized finite elements, whereas for the solid we use $\mathbb{P}_{1}$-continuous finite elements. As in in the two-dimensional test case, the time step is fixed $\delta t=10^{-4} \mathrm{~s}$ and the Nitsche and time penalty parameters to $\gamma=2500$ and $\gamma_{0}=1$. All the 3D numerical 

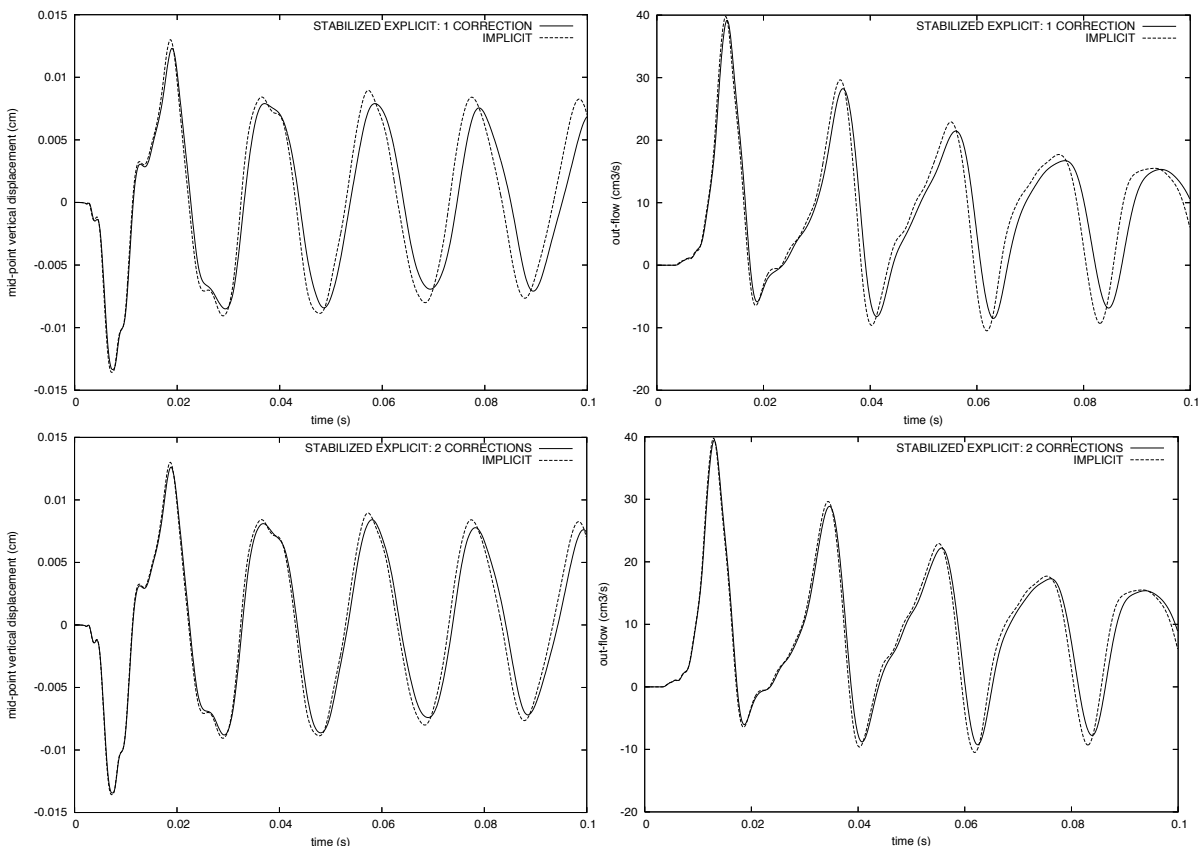

Figure 16: Stabilized explicit coupling with correction $(K=1,2, \alpha=1, \gamma=$ $\left.2500, \gamma_{0}=1\right)$. Straight vessel: interface mid-point $y$-displacement (left), and out-flow rate (right)

computations have been performed within the framework of the LifeV finite element library (www.lifev.org).

As expected, a stable pressure wave propagation is observed in both geometrical configurations (see e.g. [17, 14, 12]). Figures 12 and 13 show the fluid pressure and solid deformation (half a section) at the time instants $t=$ $0.0025,0.005,0.0075,0.01 \mathrm{~s}$. In Figures 14 to 17 we compare the interface $y$ displacements, at point $(-0.11126,-0.487464,2.5)$, and out-flow rates obtained with Algorithm 5.5 ( $\left.K=0,1,2, \alpha=1, \gamma=2500, \gamma_{0}=1\right)$, and the implicit coupling scheme (strongly enforced kinematic condition) and. The later is solved using the partitioned Newton's method proposed in [14, with an absolute displacement residual tolerance of $10^{-7}$.

Without correction, Figures 14 and 15 , the stabilized explicit scheme is able to capture the global behavior of the implicit coupling solution. However, a progressive phase deterioration is clearly visible, particularly for the curved vessel. This illustrates the impact of the optimality loss introduced by weak consistency of the stabilization term, present in the error estimate (44) with a loss of half-a-power in $\delta t$.

Figures 16 and 17 show that, as in the two-dimensional linear test case, one correction is sufficient to recover all the local features of the implicit coupling solution. In order words, it achieves first order accuracy $O(\delta t)$. Note that a progressive slightly phase deterioration is still latent, which might be related to the factor $T$ in the error estimate (44). After two correction steps the numerical solution is almost unchanged. Again, once the same order is reached, further corrections are superfluous. This feature is also illustrated in Figure 18, where 

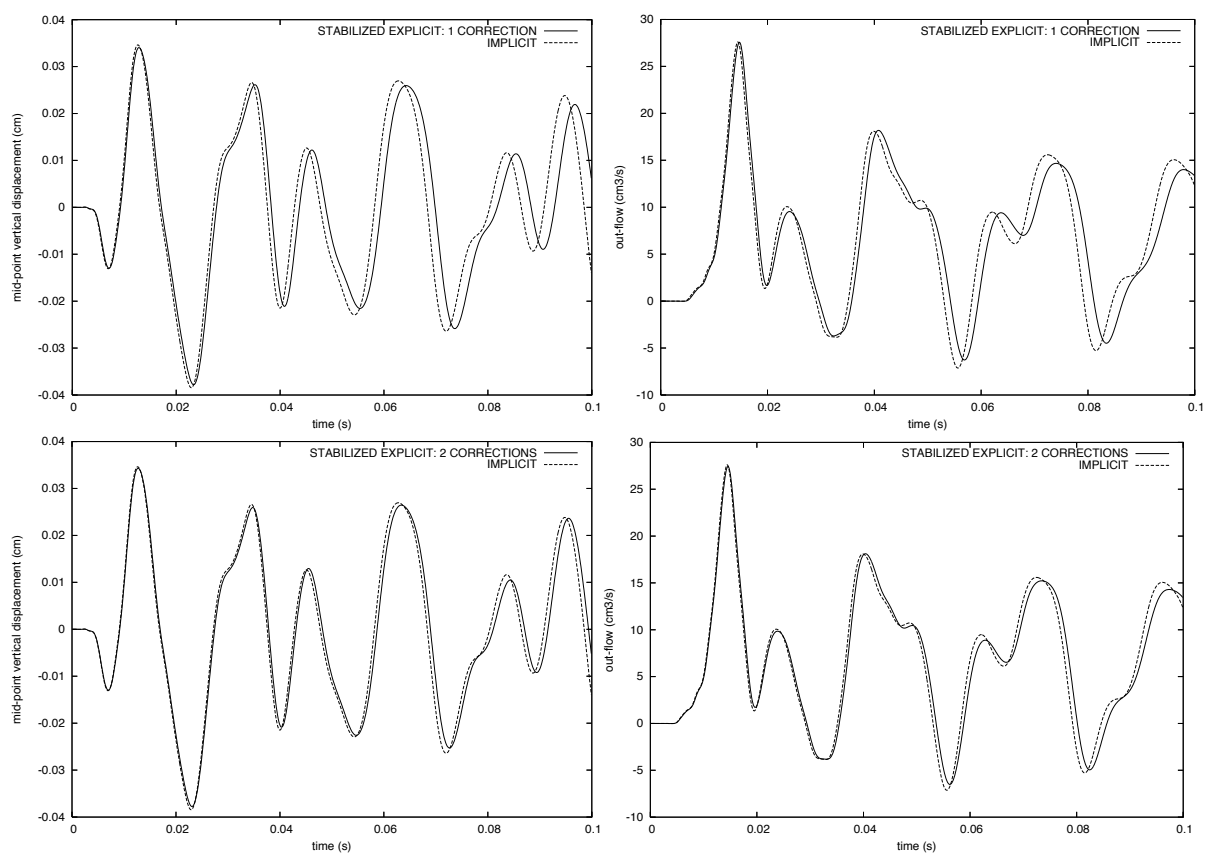

Figure 17: Stabilized explicit coupling with correction $(K=1,2, \alpha=1, \gamma=$ 2500, $\gamma_{0}=1$ ). Curved vessel: interface mid-point $y$-displacement (left), and out-flow rate (right)
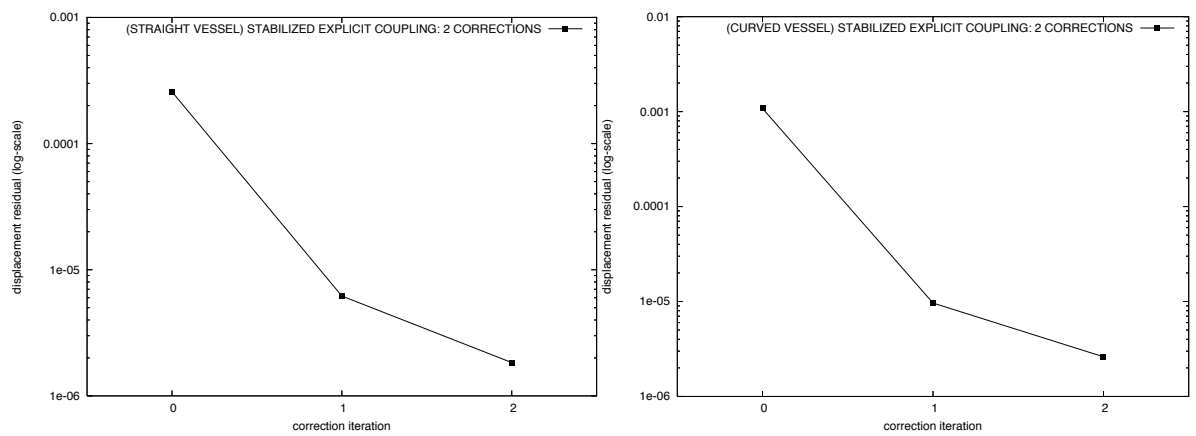

Figure 18: $L^{\infty}$-norm of the displacement residual (log-scale) at time $t=0.1 \mathrm{~s}$, stabilized explicit coupling $\left(\alpha=1, \gamma=2500, \gamma_{0}=1\right)$ with $K=2$ corrections: straight vessel (left), curved vessel (right)

we report, for the last time step, the displacement residual reduction of each correction iteration.

Finally, in Table 3 we give the elapsed (dimensionless) CPU time for both schemes in the case of the curved vessel. We notice that the explicit coupling is 8 times faster than the implicit coupling (involving an average of two Newton iterations per time step). Obviously, this difference in performance is expected to increase when dealing, for instance, with more complex geometries. 


\begin{tabular}{|c|c|}
\hline COUPLING & CPU time \\
\hline Implicit & 8 \\
\hline $\begin{array}{c}\text { Stabilized explicit } \\
\text { correction }\end{array}$ & 1 \\
\hline
\end{tabular}

Table 3: Elapsed CPU time (dimensionless): 1000 time steps of length $\delta t=$ $10^{-4} s$

\section{Conclusion}

In the present paper we have considered the efficient numerical solution of fluidstructure interaction problems involving a viscous incompressible fluid. In such framework, the standard explicit coupling procedures are known to give rise to numerical stabilities in presence of a strong added-mass effect: fluid-structure density ratio and geometry of the domain (see e.g. 7, 16). For a linear model problem, we have proved that, if $\delta t=O(h)$ and the free (dimensionless) parameters $\gamma$ and $\gamma_{0}$ are large enough, the proposed explicit coupling scheme is stable irrespectively of the amount of added-mass effect in the system. Numerical experiments confirmed this theoretical stability result. The key ingredients for the stability of the method are:

- the Nitsche treatment of the interface coupling conditions,

- the addition of a weakly consistent penalization of the (time) fluid pressure fluctuations at the interface.

The method is flexible with respect to the choice of time stepping schemes, for the fluid and the structure, and allows for independent meshing of both domains.

As regards accuracy, the order of the scheme is expected to be $O\left(h^{\frac{1}{2}} \delta t^{\frac{1}{2}}\right)$, due to the weak consistency of the stabilization term. Note that, since $\delta t=$ $O(h)$ for stability, the obtained convergence rate is optimal if a piece-wise linear approximation is used in space. In practice, however, the scheme may suffer from a deterioration of the precision in time, which is $\mathrm{O}\left(\delta t^{\frac{1}{2}}\right)$ in a fixed mesh. We have proposed the enhance the time accuracy of the scheme by using a defect-correction approach. Numerical experiments, in the 2D linear and the 3D non-linear cases, have shown that one correction step allows to recover the $O(\delta t)$ accuracy of the underlying implicit coupling scheme.

The preliminary numerical results, reported in this paper, indicate that the proposed coupling strategy provides a simple and robust approach to the explicit time-stepping of fluid-structure interaction problems, involving a viscous incompressible fluid.

\section{Acknowledgments}

We thank Ido Akkerman and Kris van der Zee for an inspiring discussion at the WCCM/ECCOMAS '08 conference that led to further understanding of the explicit coupling scheme. 


\section{References}

[1] S. Badia, F. Nobile, and C. Vergara. Fluid-structure partitioned procedures based on Robin transmission conditions. J. Comp. Phys., 227:7027-7051, 2008.

[2] K.J. Bathe and H. Zhang. Finite element developments for general fluid flows with structural interactions. Int. J. Num. Meth. Engng., 2004.

[3] R. Becker, P. Hansbo, and R. Stenberg. A finite element method for domain decomposition with non-matching grids. M2AN Math. Model. Numer. Anal., 37(2):209-225, 2003.

[4] E. Burman and M.A. Fernández. Stabilized explicit coupling for fluidstructure interaction using Nitsche's method. C. R. Math. Acad. Sci. Paris, 345(8):467-472, 2007.

[5] E. Burman and P. Hansbo. A unified stabilized method for Stokes' and Darcy's equations. J. Comput. Appl. Math., 198:35-51, 2007.

[6] E. Burman and P. Zunino. A domain decomposition method based on interior penalties for advection-diffusion-reaction problems. Siam Jour. Num. Anal., 44:1612-1638, 2006.

[7] P. Causin, J.-F. Gerbeau, and F. Nobile. Added-mass effect in the design of partitioned algorithms for fluid-structure problems. Comput. Methods Appl. Mech. Engrg., 194(42-44):4506-4527, 2005.

[8] S. Deparis, M. Discacciati, G. Fourestey, and A. Quarteroni. Fluidstructure algorithms based on Steklov-Poincaré operators. Comput. Methods Appl. Mech. Engrg., 195(41-43):5797-5812, 2006.

[9] S. Deparis, M.A. Fernández, and L. Formaggia. Acceleration of a fixed point algorithm for fluid-structure interaction using transpiration conditions. M2AN Math. Model. Numer. Anal., 37(4):601-616, 2003.

[10] J. Donéa, S. Giuliani, and J. P. Halleux. An arbitrary Lagrangian-Eulerian finite element method for transient dynamic fluid-structure interactions. Comp. Meth. Appl. Mech. Engng., pages 689-723, 1982.

[11] C. Farhat, K. van der Zee, and Ph. Geuzaine. Provably second-order timeaccurate loosely-coupled solution algorithms for transient nonlinear aeroelasticity. Comput. Methods Appl. Mech. Engrg., 195(17-18):1973-2001, 2006.

[12] M.A. Fernández, J.F. Gerbeau, and C. Grandmont. A projection semiimplicit scheme for the coupling of an elastic structure with an incompressible fluid. Int. J. Num. Meth. Engrg., 69(4):794-821, 2007.

[13] M.A. Fernández and M. Moubachir. An exact block-Newton algorithm for solving fluid-structure interaction problems. C. R. Math. Acad. Sci. Paris, 336(8):681-686, 2003. 
[14] M.A. Fernández and M. Moubachir. A Newton method using exact Jacobians for solving fluid-structure coupling. Comp. \& Struct., 83:127-142, 2005.

[15] L. Formaggia, J.-F. Gerbeau, F. Nobile, and A. Quarteroni. On the coupling of 3D and 1D Navier-Stokes equations for flow problems in compliant vessels. Comp. Meth. Appl. Mech. Engrg., 191(6-7):561-582, 2001.

[16] C. Förster, W.A. Wall, and E. Ramm. Artificial added mass instabilities in sequential staggered coupling of nonlinear structures and incompressible viscous flows. Comput. Methods Appl. Mech. Engrg., 196(7):1278-1293, 2007.

[17] J.-F. Gerbeau and M. Vidrascu. A quasi-Newton algorithm based on a reduced model for fluid-structure interactions problems in blood flows. Math. Model. Num. Anal., 37(4):631-648, 2003.

[18] P. Hansbo. Nitsche's method for interface problems in computational mechanics. GAMM-Mitt., 28(2):183-206, 2005.

[19] P. Hansbo and J. Hermansson. Nitsche's method for coupling non-matching meshes in fluid-structure vibration problems. Computational Mechanics, 32(1-2):134-139, 2003.

[20] P. Hansbo, J. Hermansson, and T. Svedberg. Nitsche's method combined with space-time finite elements for ALE fluid-structure interaction problems. Comput. Methods Appl. Mech. Engrg., 193:4195-4206, 2004.

[21] F. Hecht, O. Pironneau, A. Le Hyaric, and K. Ohtsuka. FreeFem $++v$. 2.11. User's Manual. University of Paris 6.

[22] E. Järvinen, P. Räback, and M. Lyly. Optimization of fluid-structure interaction scheme for arterial flow simulations. In 2nd International Symposium on Modelling of Physiological Flows - MPF 2005, Sesimbra, Portugal, 2005.

[23] W. Layton, H. K. Lee, and J. Peterson. A defect-correction method for the incompressible Navier-Stokes equations. Appl. Math. Comput., 129(1):119,2002 .

[24] P. Le Tallec and J. Mouro. Fluid structure interaction with large structural displacements. Comput. Meth. Appl. Mech. Engrg., 190:3039-3067, 2001.

[25] D. P. Mok and W. A. Wall. Partitioned analysis schemes for the transient interaction of incompressible flows and nonlinear flexible structures. In K. Schweizerhof W.A. Wall, K.U. Bletzinger, editor, Trends in computational structural mechanics, Barcelona, 2001. CIMNE.

[26] D. P. Mok, W. A. Wall, and E. Ramm. Partitioned analysis approach for the transient, coupled response of viscous fluids and flexible structures. In W. Wunderlich, editor, Proceedings of the European Conference on Computational Mechanics. ECCM'99, TU Munich, 1999.

[27] D. P. Mok, W. A. Wall, and E. Ramm. Accelerated iterative substructuring schemes for instationary fluid-structure interaction. In K.J. Bathe, editor, Computational Fluid and Solid Mechanics, pages 1325-1328. Elsevier, 2001. 
[28] H. Morand and R. Ohayon. Fluid-Structure Interaction: Applied Numerical Methods. John Wiley \& Sons, 1995.

[29] J. Nitsche. über ein Variationsprinzip zur Lösung von Dirichlet-Problemen bei Verwendung von Teilräumen, die keinen Randbedingungen unterworfen sind. Abh. Math. Sem. Univ. Hamburg, 36:9-15, 1971.

[30] F. Nobile. Numerical approximation of fluid-structure interaction problems with application to haemodynamics. PhD thesis, EPFL, Switzerland, 2001.

[31] K.C. Park, C.A. Felippa, and J.A. Deruntz. Stabilization of staggered solution procedures for fluid-structure interaction analysis. In Belytschko. T. and T.L. Geers, editors, Computational Methods for Fluid-Structure Interaction Problems, volume 26, pages 95-124. American Society of Mechanical Engineers, 1977.

[32] S. Piperno. Simulation numérique de phénomènes d'interaction fluidestructure. PhD thesis, Ecole Nationale des Ponts et Chaussées, 1995.

[33] S. Piperno. Explicit/implicit fluid/structure staggered procedures with a structural predictor and fluid subcycling for 2D inviscid aeroelastic simulations. Internat. J. Numer. Methods Fluids, 25(10):1207-1226, 1997.

[34] A. Quaini and A. Quarteroni. A semi-implicit approach for fluid-structure interaction based on an algebraic fractional step method. Math. Models Methods Appl. Sci., 17(6):957-983, 2007.

[35] P. Räback, J. Ruokolainen, M. Lyly, and E. Järvinen. Fluid-structure interaction boundary conditions by artificial compressibility. In ECCOMAS Computational Fluid Dynamics Conference, Swansea, UK, 2001.

[36] K. Riemslagh, J.A. Vierendeels, and E. Dick. Coupling of a Navier-Stokes solver and an elastic boundary solver for unsteady problems. In Computational Fluid Dynamics '98: Proceeding of the Fourth European Computational fluid Dynamics Conference, volume 2, pages 1040-1045, 1998.

[37] H.J. Stetter. The defect correction principle and discretization methods. Numer. Math., 29:425-443, 1978.

[38] T.E. Tezduyar. Finite element methods for fluid dynamics with moving boundaries and interfaces. Arch. Comput. Methods Engrg., 8:83-130, 2001.

[39] V. Thomée. Galerkin finite element methods for parabolic problems, volume 25 of Springer Series in Computational Mathematics. Springer-Verlag, Berlin, second edition, 2006.

[40] J.A. Vierendeels, K. Riemslagh, E. Dick, and P.R. Verdonck. Computer simulation of intraventricular ow and pressure gradients during diastole. $J$. Biomech. Eng. Trans. ASME 2000, 122(6):667-674, 2000.

\section{Contents}

1 Introduction 
\begin{tabular}{|lll}
2 & A simplified coupled problem & 4
\end{tabular}

3 Nitsche's formulation: space semi-discretization 5

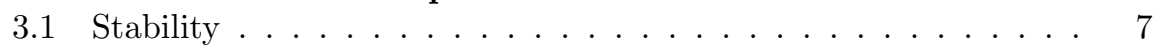

3.2 Partitioned formulation $\ldots \ldots \ldots \ldots \ldots \ldots$

\begin{tabular}{|lll}
4 & Time discretization: coupling strategies & 9
\end{tabular}

4.1 Implicit coupling . . . . . . . . . . . . . . . . . . . . 10

$4.1 .1 \quad$ Stability analysis - implicit coupling . . . . . . . . . . 11

4.2 Explicit coupling . . . . . . . . . . . . . . . . . 11

$4.2 .1 \quad$ Stability analysis - explicit coupling . . . . . . . . . 13

\begin{tabular}{|lll}
5 & Stabilized explicit coupling & 15
\end{tabular}

5.1 Stability analysis $\ldots \ldots \ldots \ldots \ldots \ldots \ldots$. . . . . . . . . 17

$5.2 \quad$ Stabilization and artificial compressibility . . . . . . . . . . . 19

5.3 Weak consistency and error estimates . . . . . . . . . . . . 20

5.4 Improving time accuracy: defect-correction iterations . . . . . . . 22

5.5 The non-linear case . . . . . . . . . . . . . . . . 24

$\begin{array}{lll}6 & \text { Numerical experiments } & 27\end{array}$

6.1 A linear two-dimensional test case . . . . . . . . . . . . . 27

6.2 A non-linear three-dimensional test case . . . . . . . . . . . . 32

\begin{tabular}{lll}
\hline 7 Conclusion & 37
\end{tabular} 
Unité de recherche INRIA Rocquencourt Domaine de Voluceau - Rocquencourt - BP 105 - 78153 Le Chesnay Cedex (France)

Unité de recherche INRIA Futurs : Parc Club Orsay Université - ZAC des Vignes 4, rue Jacques Monod - 91893 ORSAY Cedex (France)

Unité de recherche INRIA Lorraine : LORIA, Technopôle de Nancy-Brabois - Campus scientifique 615, rue du Jardin Botanique - BP 101 - 54602 Villers-lès-Nancy Cedex (France)

Unité de recherche INRIA Rennes : IRISA, Campus universitaire de Beaulieu - 35042 Rennes Cedex (France)

Unité de recherche INRIA Rhône-Alpes : 655, avenue de l'Europe - 38334 Montbonnot Saint-Ismier (France) Unité de recherche INRIA Sophia Antipolis : 2004, route des Lucioles - BP 93 - 06902 Sophia Antipolis Cedex (France) 